\title{
I Crisomelidi (Coleoptera; Chrysomelidae) del Parco di Montevecchia e della Valle del Curone (Italia, Lombardia, provincia di Lecco)
}

\author{
Laura Farina
}

\begin{abstract}
Riassunto - Vengono presentati i risultati di un'indagine faunistica condotta nel Parco Regionale di Montevecchia e della Valle del Curone, localizzato all'estremità meridionale del sistema Sudalpino, in provincia di Lecco. Viene fornito un elenco delle 92 specie di Crisomelidi censite (tre delle quali risultano nuove per la fauna lombarda: Aphthona sicelidis, Dibolia femoralis, Crepidodera fulvicornis) con indicazioni corologiche, fenologiche e la lista delle piante nutrici, ricavate in parte dalla letteratura e in parte da osservazioni sul campo. Vengono inoltre fornite alcune considerazioni sistematiche e biogeografiche relative alla specie Aphthona sicelidis. Le raccolte sono state effettuate prevalentemente in 9 stazioni situate all'interno del parco regionale e selezionate in base a criteri fitosociologico-vegetazionali. L'analisi zoogeografica mostra che il gruppo corologico maggiormente rappresentato è quello delle specie ad ampia distribuzione nella regione Olartica ( 57 specie $=62 \%$ ), seguito dagli elementi ad ampia distribuzione in Europa ( 26 specie $=28 \%$ ), e nel bacino mediterraneo $(5$ specie $=6 \%)$; i gruppi delle specie afrotropicali e orientali presenti anche nell'area mediterranea sono rappresentati da una sola specie (Chaetocnema hortensis), i gruppi di origine Neartica invece sono rappresentati da tre specie (Diabrotica virgifera, Ophraella communa, Leptinotarsa decemlineata).
\end{abstract}

Parole chiave: Chrysomelidae, Lombardia, Parco di Montevecchia e della Valle del Curone, fenologia, zoogeografia, tassonomia.

\begin{abstract}
Leaf beetles (Coleoptera Chrysomelidae) from Montevecchia and Curone Valley Natural Park (Italy, Lombardy, Lecco).

This paper is the result of a faunistic research on the Chrysomelidae fauna of Montevecchia and Curone Valley Natural Park. The Park is located south of the Alps, in Lombardy, in the province of Lecco. The author provides a list of 92 species collected. Three of these are new for the Lombardy fauna: Aphthona sicelidis, Dibolia femoralis and Crepidodera fulvicornis. The paper contains chorological and phenological notes for many species and taxonomical remarks on Aphthona sicelidis. Most specimens have been collected in 9 sampling-sites placed inside the Park and selected according to physiognomic-vegetational criteria. The zoogeographical analysis shows that the list of species is dominated by Olarctic species (57 species $=62 \%)$, followed by European elements $(26$ species $=28 \%)$ and Mediterranean species ( 5 species $=6 \%$ ). Afrotropical and Oriental species also present in the Mediterranean area are represented by a single species (Chaetocnema hortensis), the groups of Nearctic
\end{abstract}

Via A. Stoppani 17/A, 23880 Casatenovo (Lc), Italia

E-mail: lau.farina@libero.it

(C) 2015 Laura Farina

Received: 1 April 2015

Accepted for publication: 2 September 2015 origin instead are represented by three species (Diabrotica virgifera, Ophraella communa, Leptinotarsa decemlineata).

Key words: Chrysomelidae, Lombardy, Montevecchia and Curone Valley Park, Phenology, Geographic distribution, Taxonomy.

\section{INTRODUZIONE}

La zona prealpina delle province di Lecco e Como è stata ed è attualmente oggetto di intense attività di studio della fauna crisomelidologica. Le zone indagate sono state: il lago di Sartirana (Brivio, 1970), il Monte Barro (Leonardi \& Sassi, 1997), il Triangolo Lariano (Sassi, 2007). Sono attualmente in corso indagini sui Crisomelidi del gruppo delle Grigne ad opera di Renato Regalin.

Questo lavoro rappresenta il primo contributo alla conoscenza dei Coleotteri Crisomelidi del Parco di Montevecchia e della Valle del Curone, il cui territorio è situato in Provincia di Lecco, a Sud dei primi rilievi prealpini.

Le campagne di ricerca nel Parco hanno fruttato la raccolta di 1852 esemplari appartenenti a 92 specie, alcune di particolare interesse, inedite per la Lombardia (Aphthona sicelidis Weise, Crepidodera fulvicornis F., Dibolia femoralis Redtenbacher).

Il sistema collinare del Parco ospita una copertura prevalentemente forestale. Sui versanti a esposizione meridionale la copertura forestale ha lasciato spazio a vigneti e frutteti, mentre nelle zone di fondovalle sono diffusi prati e semilavorativi. Su alcuni versanti terrazzati con esposizione meridionale, si individuano aree incolte derivanti dal progressivo abbandono delle pratiche colturali. Questi rilievi presentano un mosaico di prati magri intercalati da nuclei arbustati e arborati più o meno estesi (Fig. 1). Le principali formazioni prative del Parco, oggetto delle raccolte di Crisomelidi, possono essere attribuite alle seguenti unità fitosociologiche (Panseri, 2000):

Arrhenatheretea: rappresenta la comunità di prato regolarmente falciato, di condizione meso-eutrofiche, con suolo profondo, fertile, pianeggiante. Tra le specie vegetali che abbondano in questa tipologia di prato, Rumex acetosa, Ranunculus acris, Trifolium pratense, Galium mollugo, Salvia pratensis, Plantago lanceolata, Dactylis glomerata, Arrhenatherum elatius, Silene vulgaris. 


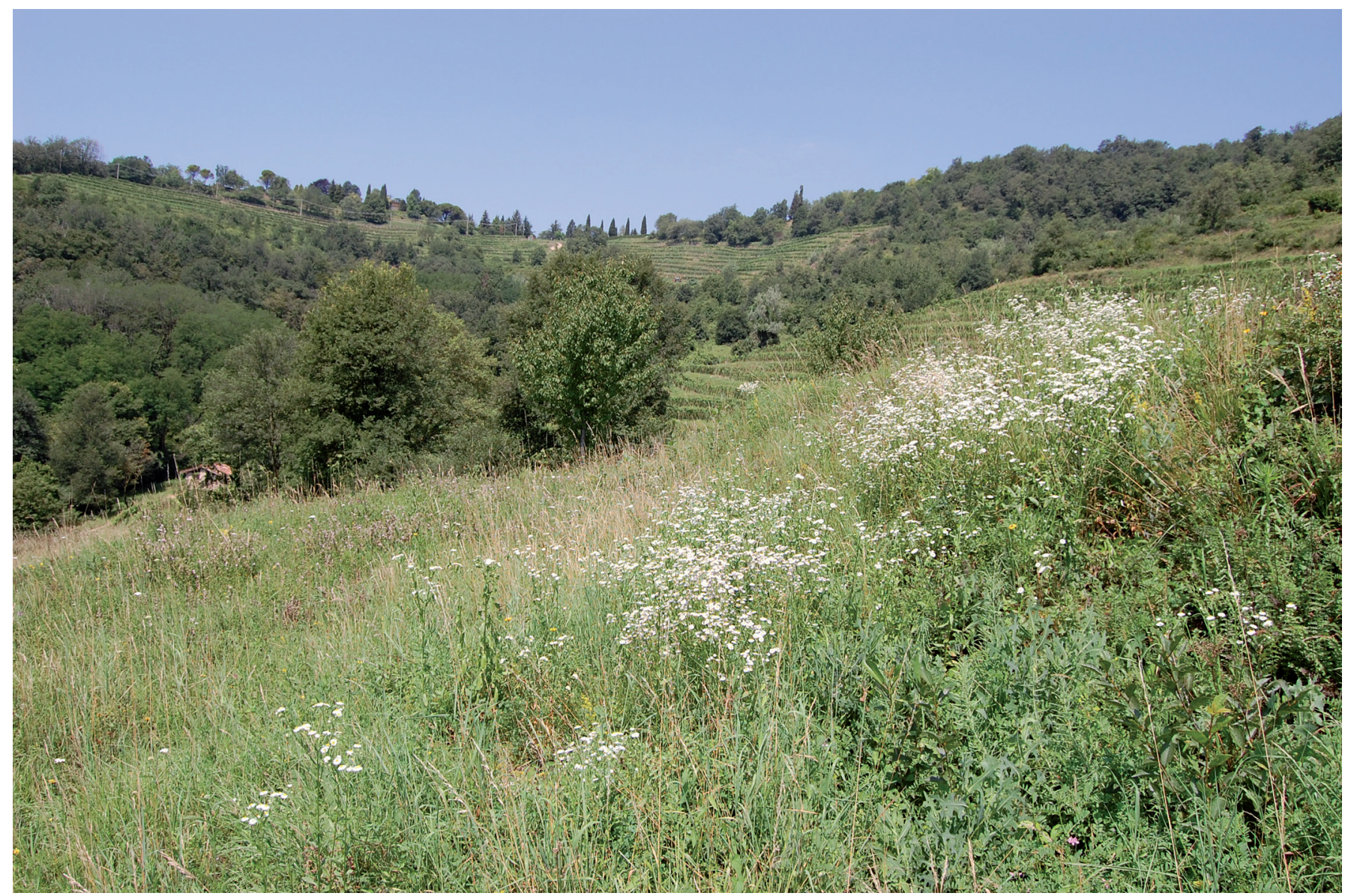

Fig. 1 - Prati magri di Perego. (Foto L. Farina).

Festuco-Brometea (in particolare specie dei Brometalia e Mesobromion): si tratta dei prati magri regolarmente falciati o per lo meno falciati in tempi relativamente recenti e sono caratterizzati da elementi floristici sub mediterranei e sud europei. Tra le specie caratteristiche si trovano $G a$ lium verum, Koeleria cristata, Bothriochloa ischaemum, Filipendula vulgaris, Salvia pratensis, Prunella grandiflora, Stachys recta, Odontites luteus, Poterium sanguisorba (Festuco-Brometea), Hippocrepis comosa, Scabiosa portae (Brometalia), Ononis spinosa (Mesobromion). In condizioni di minore aridità edafica, alle essenze dei Festuco-Brometea si associano specie caratteristiche dei prati falciati (Arrhenatherion): Arrhenatherum elatius, Achillea roseoalba, Centaurea nigrescens. In aree caratterizzate da substrato affiorante, e quindi con suolo più sottile, compaiono specie caratteristiche di Xerobromion: Teucrium montanum e Globularia bisnagarica.

Trifolio-Geranetea: rappresentano le comunità erbacee ecotonali di margine boschivo e sono in stretta relazione con le cenosi forestali. Sono sempre presenti Aster amellus e Cervaria rivini, specie caratteristiche del GeranioPeucedatenum cervariae.

Molinietalia: questi ambienti, situati nelle aree con ristagni di acqua stagionale, sono colonizzati da una robusta graminacea, la Molinia coerulea. Tra le specie osservabili: Lythrum salicaria, Filipendula ulmaria, Valeriana officinalis.

\section{MATERIALI E METODI}

La ricerca si basa su raccolte qualitative pluriennali svolte dall'autrice nel Parco. Le raccolte sono state effettuate con il retino da sfalcio e con l'utilizzo dell'ombrello entomologico in 9 stazioni prative. Alcune specie sono state raccolte "a vista" sulle piante oppure deambulanti al suolo. All'interno delle stazioni le raccolte sono state condotte da marzo a novembre, cercando di mantenere una certa uniformità nella durata dei campionamenti.

Il materiale raccolto è conservato a secco nella collezione dell'autrice. Sono state incluse nel lavoro alcune considerazioni sistematiche e biogeografiche relative a Aphthona sicelidis e considerazioni biogeografiche su Crepidodera fulvicornis e Dibolia femoralis. A questo proposito sono stati esaminati anche materiali reperiti presso collezioni museali e private (Tab. 1).

Tab. 1 - Collezioni esaminate e sigle di riferimento.

\begin{tabular}{|l|l|}
\hline Sigla & Collezione \\
\hline $\mathrm{MBa}$ & Naturhistorisches Museum Basel \\
\hline $\mathrm{MMi}$ & Museo Civico di Storia Naturale di Milano \\
\hline $\mathrm{CFa}$ & Laura Farina (Casatenovo, Lecco) \\
\hline $\mathrm{CMt}$ & Fernando Montemurro (Taranto) \\
\hline $\mathrm{CZo}$ & Stefano Zoia (Milano) \\
\hline
\end{tabular}


Le raccolte sono state effettuate prevalentemente in 9 stazioni prative (Fig. 2), selezionate in base a criteri fitosociologico-vegetazionali. L'elenco delle stazioni è illustrato in Tab. 2

L'elenco sistematico è stato redatto facendo riferimento alla Checklist delle specie della fauna italiana (Biondi et al., 1994), mentre per la nomenclatura si è fatto riferimento soprattutto alla Fauna Europaea (Biondi, 2013). La tasso-

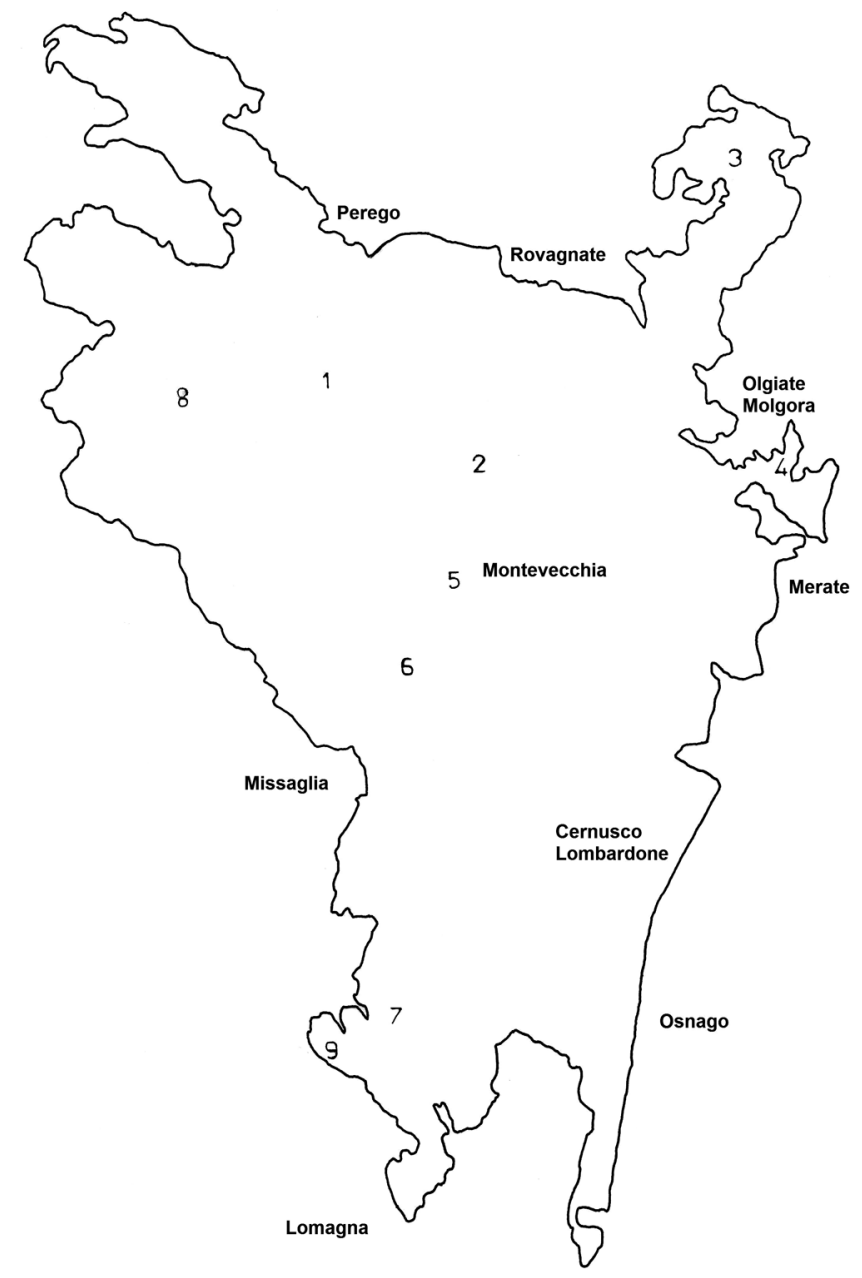

Fig. 2 - Carta del Parco con ubicazione delle stazioni di raccolta. nomia segue il Catalogue of Palaearctic Coleoptera (Löbl \& Smetana, 2010). Le indicazioni relative al corotipo di appartenenza delle singole specie sono state individuate secondo i criteri proposti da Vigna Taglianti et al. (1999).

\section{Area di indagine}

Il Parco Regionale di Montevecchia e della Valle del Curone (Fig. 3) coincide con l'estremo lembo verde della Brianza sud-orientale (Lombardia, Italia). Il territorio della Brianza è compreso tra le Prealpi a Nord, l'Adda ad Est, il Lambro ad Ovest ed i piani ferrettizzati a Sud, fino alla latitudine di Arcore (Ronzoni, 1998). Il Parco, istituito dalla Legge Regionale n. 77 del 30/11/1983, si presenta come un'area molto diversificata, caratterizzata da zone di rilevante interesse ambientale (sorgenti petrificanti, prati magri, boschi igrofili) accanto a centri urbani, insediamenti produttivi, aree destinate all'agricoltura e all'allevamento. Il Parco ricopre una superficie di circa 2741 ettari e il suo territorio interessa in tutto o in parte undici Comuni della Provincia di Lecco: Cernusco Lombardone, Lomagna, Merate, Missaglia, Montevecchia, Olgiate Molgora, Osnago, Perego, Rovagnate, Sirtori, Viganò.

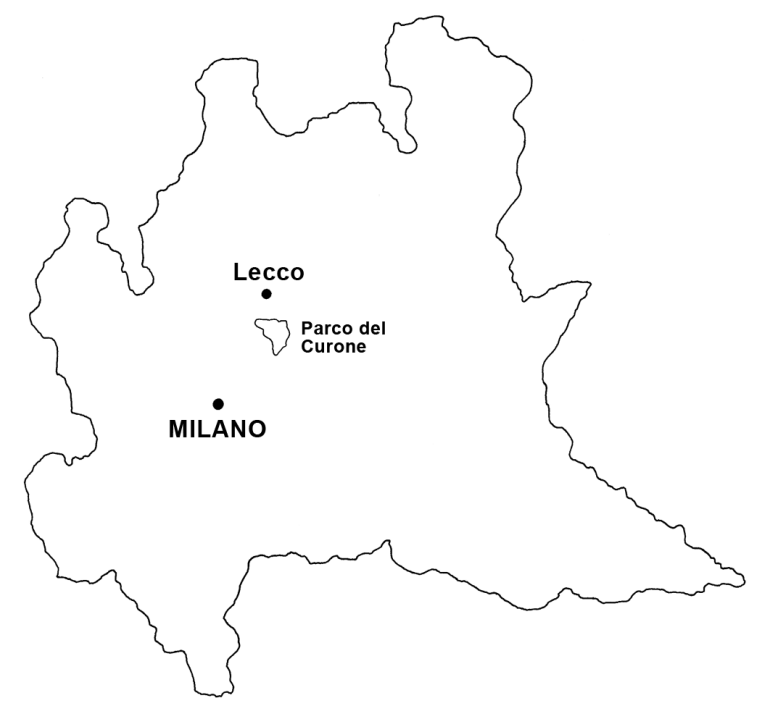

Fig. 3 - Localizzazione geografica del Parco in Lombardia.

Tab. 2 - Elenco delle stazioni in cui è stata divisa l'area di studio.

\begin{tabular}{|c|c|c|c|c|}
\hline N. della stazione & Abbreviazione & Stazione & Media altitudinale & Coordinate geografiche \\
\hline 1 & Per & Perego & $340 \mathrm{~m}$ & 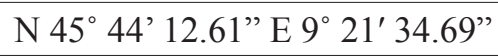 \\
\hline 2 & Rov & Rovagnate & $370 \mathrm{~m}$ & N 45 44'16.83" E 9²2’09.57” \\
\hline 3 & Mon & Mondonico & $330 \mathrm{~m}$ & N 45 44'02.84" E 9०23'28.36" \\
\hline 4 & $\mathrm{Cal}$ & Calendone & $290 \mathrm{~m}$ & 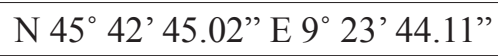 \\
\hline 5 & Cas & Cà del Soldato & $310 \mathrm{~m}$ & $\mathrm{~N} 45^{\circ} 42^{\prime} 48.71^{\prime \prime} \mathrm{E} 9^{\circ} 22^{\prime} 17.80^{\prime \prime}$ \\
\hline 6 & Ver & Vertaggera & $320 \mathrm{~m}$ & $\mathrm{~N} 45^{\circ} 42^{\prime} 11.58^{\prime \prime}$ E 9०22'47.51" \\
\hline 7 & Squ & Squallera & $270 \mathrm{~m}$ & $\mathrm{~N} 45^{\circ} 40^{\prime} 33.18^{\prime \prime}$ E 9०22'57.21" \\
\hline 8 & Vsc & Valle S. Croce & $330 \mathrm{~m}$ & N 45 43'08.66" E 9²0'46.78" \\
\hline 9 & Lan & Landriano & $273 \mathrm{~m}$ & $\mathrm{~N} 45^{\circ} 40^{\prime} 31.73^{\prime \prime}$ E $9^{\circ} 21^{\prime} 28.28^{\prime \prime}$ \\
\hline
\end{tabular}


L'area oggetto di studio è situata dal punto di vista geologico all'estremità meridionale del sistema Sudalpino. Al suo interno è possibile operare una distinzione tra il settore settentrionale e quello meridionale. Nel primo, rappresentato dal crinale che unisce Montevecchia a Lissolo e dalle due valli ad esso laterali, la Valle di Santa Croce e la Valle del Curone, affiora principalmente il substrato lapideo, costituito da rocce di età cretacico-eocenica, per lo più calcareniti e calcari. In particolare è possibile riconoscere le formazioni del Flysch di Bergamo e della Scaglia Cinerea e Rossa. Nel secondo affiorano invece estesamente depositi quaternari di origine glaciale. I principali tipi di suolo presenti sono costituiti dal ferretto, che occupa le zone terrazzate meridionali, e dal suolo basico con abbondanti ossidi di calcio, alluminio e ferro ed elementi argillosi e silicatici (Previati, 1997). Questa diversa origine geologica differenzia anche morfologicamente le due zone conferendo al settore settentrionale un aspetto più aspro, con valli abbastanza incise e versanti ripidi. Nella zona meridionale l'estesa copertura di depositi sciolti di origine glaciale conferisce al territorio un aspetto più dolce, con valli ampie dal fondo pianeggiante e con una caratteristica serie di blande ondulazioni di limitata altezza e versanti poco inclinati che lasciano il posto verso sud ad aree decisamente pianeggianti. L'assetto strutturale e le caratteristiche geologiche della zona ne determinano strettamente le condizioni idrogeologiche. Nella parte collinare di affioramento del substrato prequaternario, ove sono frequenti intercalazioni marnose poco permeabili, l'intenso stato di fratturazione consente la venuta a giorno delle acque percolanti nei livelli più permeabili; in questo contesto si inquadrano le sorgenti del torrente Molgoretta in alta Valle S. Croce e del torrente Curone nella valle omonima. Nelle zone di pianura invece, la elevata permeabilità dei depositi fluvioglaciali di fondovalle consente l'instaurarsi di una falda idrica, alimentata direttamente dalle precipitazioni, posta ad una profondità di 15-20 metri (Previati, 1997) e caratterizzata da un'elevata escursione, in funzione dell'entità delle precipitazioni.

Il sistema collinare del Parco ospita una copertura prevalentemente forestale. Il complesso boscato Valle del Curone - Valle S. Croce - Viganò rappresenta 1'ultima superficie forestale di considerevoli dimensioni in continuità con le formazioni boscate dei rilievi prealpini.

All'interno del Parco è stato individuato un Sito di Interesse Comunitario (SIC) che occupa una superficie totale di 1350 ha. Il sito include totalmente la Riserva Naturale della Valle Santa Croce e dell'Alta Valle del Curone, classificata quale riserva orientata paesistico-forestale. L'importanza del sito risiede nella presenza di alcune specie ed habitat di importanza comunitaria, quali: sorgenti petrificanti (Cratoneurion), prati magri (Festuco-Brometalia), boschi igrofili (Alno-Ulmion) (AA.VV., 2010).

\section{RISULTATI}

Per ogni stazione ho indicato le specie esclusive (specificità) e le tre specie più comuni con il relativo indice di dominanza (sensu Berger e Parker, 1970). Si tratta di un indice che indica il numero di esemplari di ogni specie sul totale di esemplari raccolti.
Stazione 1: località Perego, esposizione S. L'area è caratterizzata dalla presenza di praterie terrazzate semiaride, regolarmente falciate. Alcuni terrazzamenti sono destinati alla coltivazione della vite.

Specificità: Lema cyanella (Linné, 1758), Oulema mauroi (Bezděk \& Baselga, 2015), Chrysolina marginata (Linné,1758), Luperus flavipes (Linné, 1767), Phyllotreta punctulata (Marsham,1802), Aphthona ovata Foudras, 1860, Longitarsus codinai Madar \& Madar, 1965, Longitarsus helvolus Kutschera, 1864, Dibolia femoralis Redtenbacher, 1849, Cassida prasina Illiger, 1798. Dominanze: Longitarsus pratensis (Panzer, 1794) (0,213), Sphaeroderma rubidum (Graëlls, 1858) (0,108), Cryptocephalus flavipes Fabricius, 1781 (0,092). 48 specie censite; 314 esemplari.

Stazione 2: località Monte di Rovagnate, esposizione dei terrazzamenti parte a N-O e parte a S. L'area è caratterizzata da praterie semiaride, disposte su due serie di terrazzamenti. I prati magri, non più gestiti, sono intercalati a nuclei arbustati e arborati. La ricostruzione della copertura forestale è testimoniata dalla presenza di rovo, roverella, corniolo, felce aquilina.

Specificità: Timarcha nicaeensis Villa, 1835, Psylliodes cuprea (Koch, 1803), Labidostomis humeralis (Schneider, 1792), Labidostomis lucida (Germar, 1824), Pachybrachis tesselatus (Olivier, 1791), Cryptocephalus labiatus (Linné, 1761). Dominanze: Aphthona herbigrada (Curtis, 1837) (0,147), Longitarsus salviae Gruev, 1975 (0,109), Cryptocephalus flavipes $(0,105)$. 38 specie censite; 258 esemplari.

Stazione 3: località Mondonico di Olgiate Molgora, esposizione S-E. In quest'area si evidenzia la presenza di tre aspetti fondamentali: il fragmiteto, residui di boscaglia ripariale (Salicion cinereae), prati falciabili con elementi di prato umido (Lythrum salicaria, Filipendula ulmaria, Molinia coerulea, Equisetum spp.). In corrispondenza del corso d'acqua e dello stagno, ormai quasi del tutto interrato, sono frequenti Typha latifolia, Phragmites australis, Juncus conglomeratus.

Specificità: Aphthona venustula (Kutschera, 1861), Labidostomis longimana (Linné, 1761), Cryptocephalus janthinus Germar, 1824, Cryptocephalus parvulus Müller, 1776. Dominanze: Phyllotreta vittula (Redtenbacher,1849) $(0,177)$, Chaetocnema hortensis (Geoffroy, 1785) $(0,161)$, Longitarsus pratensis $(0,141) .34$ specie censite; 384 esemplari.

Stazione 4: località Calendone di Merate, esposizione S-E. Si tratta di una superficie prativa a ridosso di uno stagno, lungo le cui sponde sono presenti elementi di Phragmitetum australis e boscaglia ripariale (Salicion cinereae).

Specificità: Crepidodera fulvicornis (Fabricius, 1792). Dominanze: Phyllotreta vittula $(0,200)$, Crepidodera aurata (Marsham,1802) (0,182), Crepidodera fulvicornis $(0,164) .15$ specie censite; 55 esemplari raccolti.

Stazione 5: località Cà del Soldato di Montevecchia, esposizione S-E. Prato falciabile, gestito a foraggio, con elementi di prato umido (Lythrum salicaria, Filipendula 
ulmaria, Equisetum spp.). Nelle vicinanze del prato è presente uno stagno, lungo le cui sponde c'è Phragmitetum ed elementi di Salicion cinereae.

Specificità: Chrysolina fastuosa (Scopoli, 1763), Chrysomela populi Linné, 1758, Chrysomela vigintipunctata (Scopoli, 1763), Leptinotarsa decemlineata (Say, 1824), Altica brevicollis Foudras, 1861, Cassida vibex Linné, 1767. Dominanze: Crepidodera aurata $(0,319)$, Lythraria salicariae (Paikull, 1800) $(0,144)$, Phyllotreta vittula $(0,103)$. 21 specie censite; 97 esemplari.

Stazione 6: località Vertaggera di Montevecchia, esposizione S. Superficie prativa terrazzata caratterizzata dalla convivenza di elementi di prateria semiarida, elementi di prato falciabile ed elementi di disturbo marginale.

Specificità: Chrysolina herbacea (Duftschmid, 1825), Chysolina grossa (Fabricius, 1792), Chrysolina americana (Linné, 1758), Phyllotreta atra (Fabricius, 1775), Cassida margaritacea Schaller, 1783. Dominanze: Phyllotreta vittula $(0,182)$, Longitarsus luridus (Scopoli, 1763) $(0,151)$, Longitarsus pratensis $(0,143) .33$ specie censite; 258 esemplari.

Stazione 7: località Squallera di Osnago, esposizione $\mathrm{S}-\mathrm{O}$. Prevalenza di praterie semiaride terrazzate. Elementi di prato falciabile alla base dei terrazzamenti. Il prato a foraggio è attraversato per tutta la sua lunghezza da una roggia, lungo le cui rive si trovano salice bianco, ontano nero e nocciolo. Alcuni terrazzamenti si presentano irregolarmente gestiti, per cui si evidenzia una ripresa dinamica, con la presenza costante di Rubus spp., Agrimonia eupatoria e Mentha spp.

Specificità: Crioceris duodecimpunctata (Linné, 1758), Calomicrus circumfusus (Marsham, 1802), Luperus leonardii Fogato, 1979, Phyllotreta ochripes (Curtis, 1837). Dominanze: Crepidodera aurata $(0,206)$, Phyllotreta vittula $(0,156)$, Chaetocnema conducta (Motschulski, 1838) (0,126). 29 specie censite; 301 esemplari.

Stazione 8: località Valle S. Croce di Missaglia, esposizione S-E. Convivenza di elementi di prato falciabile ed elementi di disturbo marginale. Lungo il torrente Molgoretta presenza di una piccola formazione di bosco igrofilo appartenente all'alleanza Alno-Ulmion.

Specificità: Galerucella lineola (Fabricius, 1781). Dominanze: Crepidodera aurata $(0,469)$, Galerucella lineola $(0,083)$, Longitarsus pratensis $(0,083) .16$ specie censite; 96 esemplari.

Stazione 9: località Landriano di Missaglia, esposizione S. Convivenza di elementi di prato falciabile ed elementi di disturbo marginale.

Specificità: nessuna. Dominanze: Phyllotreta vittula $(0,618)$, Longitarsus pratensis $(0,067)$, Chaetocnema conducta $(0,067) .14$ specie censite; 89 esemplari.

\section{Elenco faunistico}

Viene presentato l'elenco delle specie raccolte. Nella trattazione delle singole specie vengono fornite informazioni fenologiche, indicazioni corologiche e una lista delle piante nutrici, ricavate dalla letteratura e da osservazioni dirette. Saranno seguite da un asterisco (*) le piante ospiti presenti nel Parco. I generi sono indicati in ordine sistematico, le specie sono in ordine alfabetico. Nelle osservazioni è stato inserito l'elenco delle stazioni del Parco ove la specie è stata osservata ed in alcuni casi, per le specie raccolte in un unico esemplare, la data di cattura. Sono stati riportati dati e osservazioni più approfonditi relativamente ad alcuni taxa di particolare interesse, di cui si è inteso proporre considerazioni circa la tassonomia, l'ecologia, la distribuzione geografica e la fenologia.

Crioceris duodecimpunctata (Linné, 1758)

Corotipo: Turanico-Europeo (TUE). Introdotta in Nord America.

Presenza in Italia: tutte le regioni.

Piante ospiti: varie specie di Asparagus (Liliaceae), in particolare Asparagus officinalis*.

Osservazioni: specie reperita, in volo, in un unico esemplare sui prati magri di Squallera il 27.4.2014.

Lema cyanella (Linné, 1758)

Corotipo: Asiatico-Europeo (ASE).

Presenza in Italia: Italia settentrionale, dalla Venezia Giulia al Piemonte.

Piante ospiti: Asteraceae (Cirsium, Carduus, Silybum).

Osservazioni: specie reperita in un unico esemplare sui prati magri di Perego il 13.5.2013.

\section{Oulema duftschmidi (Redtembacher, 1874)}

Corotipo: Turanico-Europeo-Mediterraneo (TEM).

Presenza in Italia: dopo la recente separazione da $\mathrm{Ou}$ lema melanopus (Linné, 1758), la distribuzione in Italia di questa specie deve essere ancora definita. Per ora risulta nota per singole regioni continentali, peninsulari e per la Sardegna (Regalin et al., 2006).

Piante ospiti: Poaceae spontanee e coltivate.

Osservazioni: specie reperita in 4 delle stazioni di campionamento, in un totale di 17 esemplari (Landriano, Mondonico, Perego, Squallera).

\section{Oulema mauroi (Bezděk \& Baselga, 2015)}

Corotipo: Sud-Europeo (SEU)

Presenza in Italia: Lombardia, Trentino Alto Adige, Veneto.

Pianta ospite: ancora sconosciuta. Probabilmente la specie è associata a Poaceae.

Osservazioni: raccolta in unico esemplare sulle praterie xerotermiche della stazione di Perego il 18.6.2013. Si tratta di una specie xerotermofila, tipica di suoli calcarei e rocciosi, endemica di zone prealpine xerotermiche con vegetazione sub mediterranea (Bezděk \& Baselga, 2015).

Timarcha (Timarcha) nicaeensis Villa, 1835

Corotipo: S-Europeo (SEU).

Presenza in Italia: Penisola.

Piante ospiti: Rubiaceae (Galium).

Osservazioni: specie raccolta in un unico esemplare il 4.3.2015, errante lungo il sentiero a Monte di Rovagnate. 
Chrysolina (Stichoptera) rossia (Illiger, 1802)

Corotipo: S-Europeo (SEU).

Presenza in Italia: Penisola.

Piante ospiti: Scrophulariaceae (Linaria).

Osservazioni: specie reperita in 2 delle stazioni di campionamento, in un totale di 3 esemplari (Perego, Vertaggera).

Chrysolina (Fastuolina) fastuosa fastuosa (Scopoli, 1763)

Corotipo: Asiatico-Europeo (ASE).

Presenza in Italia: Penisola.

Piante ospiti: Lamiaceae dei generi Galeopsis, Lamium.

Osservazioni: specie reperita in una sola stazione, a Cà del Soldato, in un totale di 3 esemplari, su Galeopsis tetrahit*. La specie è stata ritrovata anche in altre zone del Parco, al di fuori delle stazioni studiate, sempre all'interno o in prossimità di boschi di latifoglie, sulla pianta ospite precedentemente indicata.

Chrysolina (Synerga) herbacea herbacea (Duftschmid, 1825)

Corotipo: Europeo (EUR).

Presenza in Italia: tutte le regioni, eccetto Sardegna.

Piante ospiti: Lamiaceae, prevalentemente dei generi Mentha, Calamintha, Satureja e Marrubium.

Osservazioni: specie reperita in una sola stazione, a Vertaggera, in un totale di 2 esemplari, su colonie di Mentha spicata*.

Chrysolina (Melasomoptera) grossa grossa (Fabricius, 1792)

Corotipo: Mediterraneo (WME).

Presenza in Italia: dalla Liguria ed Emilia Romagna fino alla Sicilia. Presente su Alpi e Prealpi in stazioni xerotermiche (Daccordi \& Ruffo, 2005).

Piante ospiti: Lamiaceae dei generi Mentha, Calamintha, Satureja e Origanum.

Osservazioni: specie reperita solo a Vertaggera, in un totale di 2 esemplari, su Mentha spicata*.

Chrysolina (Taeniochrysea) americana (Linné, 1758)

Corotipo: Mediterraneo (MED).

Presenza in Italia: dalla Liguria alla Sicilia (versante tirrenico) e fino all'Emilia Romagna (versante adriatico). Presente pure in Sardegna e in aree xerotermiche dell'arco alpino. In espansione attiva verso nord (Daccordi \& Ruffo, 2005).

Piante ospiti: Lamiaceae dei generi Rosmarinus, Lavandula, Salvia e Thymus.

Osservazioni: specie reperita in una sola stazione, a Vertaggera, in un totale di 14 esemplari, su Rosmarinus officinalis*.

Chrysolina (Colaphodes) haemoptera haemoptera (Linné, 1758)

Corotipo: Turanico-Europeo (TUE).

Presenza in Italia: Penisola, Sardegna.

Piante ospiti: Plantaginaceae (Plantago).
Osservazioni: specie reperita in 3 delle stazioni di campionamento, in un totale di 8 esemplari (Perego, Squallera, Vertaggera).

Chrysolina (Chalcoidea) marginata marginata

(Linné,1758)

Corotipo: Paleartico (PAL).

Presenza in Italia: Penisola e Sicilia.

Piante ospiti: Asteraceae (Achillea, Artemisia, Tanacetum, Leucanthemum).

Osservazioni: specie raccolta in 2 esemplari il 13.5.2013, a Perego.

Chrysolina (Hypericia) geminata (Paykull,1799)

Corotipo: Europeo (EUR).

Presenza in Italia: regioni settentrionali e Toscana.

Piante ospiti: monofaga su Hypericum perforatum*.

Osservazioni: specie reperita in 2 delle stazioni di campionamento, in un totale di 2 esemplari (Mondonico, Perego).

Leptinotarsa decemlineata (Say, 1824)

Corotipo: N-Americano, importata in Europa e stabilizzata.

Presenza in Italia: Penisola, Sicilia.

Piante ospiti: Solanaceae spontanee e coltivate, ma anche su Chenopodiaceae (Chenopodium), Brassicaceae (Sisymbrium) e Asteraceae (Achillea).

Osservazioni: specie reperita, in diversi esemplari, in un orto a Cà del Soldato, su Solanum tuberosum*.

Chrysomela (Chrysomela) populi Linné, 1758

Corotipo: Asiatico-Europeo (ASE).

Presenza in Italia: tutte le regioni.

Piante ospiti: Salicaceae (Populus, Salix), Ramnaceae (Rhamnus cathartica*), Betulaceae (Betula pendu$\left.l a^{*}\right)$.

Osservazioni: raccolta in diversi esemplari, adulti e larve, su Populus tremula*, a Montevecchia, lungo il sentiero degli Ulivi. La stazione di campionamento più prossima a questo sentiero viene identificata in quella di Cà del Soldato.

Chrysomela (Strickerus) vigintipunctata vigintipunctata (Scopoli, 1763)

Corotipo: Asiatico-Europeo (ASE)

Presenza in Italia: regioni settentrionali e centrali.

Piante ospiti: Salicaceae (Salix).

Osservazioni: specie rinvenuta in un singolo esemplare su Salix alba* nei pressi dei ruderi di Cascina Ospedaletto, a circa $600 \mathrm{~m}$ a N-O di Cà del Soldato.

Linaeidea aenea aenea (Linné, 1758)

Corotipo: Sibirico-Europeo (SIE).

Presenza in Italia: regioni settentrionali, Toscana, Lazio, Abruzzo e Calabria.

Piante ospiti: Betulaceae (Alnus).

Osservazioni: la specie è stata raccolta in due stazioni umide, Cà del Soldato e Valle S. Croce, in un totale di 5 esemplari, su Alnus glutinosa*. 
Galerucella (Neogalerucella) lineola lineola (Fabricius, 1781)

Corotipo: Paleartico (PAL).

Presenza in Italia: Penisola, Sicilia e Sardegna.

Piante ospiti: Betulaceae (Alnus), Salicaceae (Salix, Populus nigra*), Corylaceae (Corylus avellana*), Primulaceae (Lysimachia), Polygonaceae (Rumex).

Osservazioni: la specie è stata raccolta solo in Valle $\mathrm{S}$. Croce, in un totale di 8 esemplari, su Alnus glutinosa*.

Galerucella (Neogalerucella) pusilla (Duftschmid, 1825) Corotipo: Asiatico-Europeo (ASE)

Presenza in Italia: Penisola.

Piante ospiti: Lythraceae (Lythrum salicaria*). Indicata anche su Primulaceae (Lysimachia), Lamiaceae (Stachys palustris) e Scrophulariceae (Veronica).

Osservazioni: la specie è stata raccolta su Lythrum salicaria. E' stata reperita in 4 delle stazioni di campionamento, in un totale di 42 esemplari (Calendone, Cà del Soldato, Mondonico, Rovagnate).

Galeruca (Galeruca) pomonae pomonae (Scopoli, 1763) Corotipo: Asiatico-Europeo (ASE).

Presenza in Italia: tutte le regioni.

Piante ospiti: Asteraceae (Centaurea jacea*, Cirsium palustre, Achillea, Leontodon), Lamiaceae (Salvia pratensis*, Satureja montana, Thymus), Brassicaceae (Capsella bursa-pastoris*), Dipsacaceae (Scabiosa, Knautia arvensis*, Succisa pratensis).

Osservazioni: specie reperita in 2 delle stazioni di campionamento, in un totale di 2 esemplari (Mondonico, Perego).

Galeruca (Galeruca) tanaceti tanaceti (Linné, 1758)

Corotipo: Asiatico-Europeo (ASE).

Presenza in Italia: Penisola, Sicilia.

Piante ospiti: Asteraceae (Achillea millefolium*, Tanacetum vulgare*, Taraxacum, Centaurea, Cirsium, Stellaria), Brassicaceae (Cardamine pratensis*, Brassica napus*), Caryophyllaceae (Cerastium, Silene), Apiaceae (Torilis, Pimpinella, Daucus), Campanulaceae (Campanula trachelium*), Solanaceae (Solanum tuberosum*), Chenopodiaceae (Beta vulgaris*), Polygonaceae (Rheum), Liliaceae (Allium cepa*), Fabaceae, cereali.

Osservazioni: specie reperita in 3 delle stazioni di campionamento, in un totale di 9 esemplari (Mondonico, Perego, Rovagnate, Vertaggera).

\section{Ophraella communa Le Sage, 1986}

Corotipo: specie di origine Neartica, recentemente segnalata in Italia.

Presenza in Italia: specie di origine neartica presente in Canada, Stati Uniti e Messico che è stata accidentalmente introdotta in Cina, Corea, Giappone e Taiwan. Si tratta di una specie nuova per l'Italia e per l'Europa. Le prime segnalazioni in Lombardia risalgono al 2013 (Boriani et al., 2013).

Piante ospiti: si tratta di una specie oligofaga, associata alle Asteraceae Heliantheae, con preferenza per Ambrosia artemisiifolia*. Per questa ragione viene utilizzata in Cina, e in altri Paesi, per il controllo biologico di questa infestante. Nel Parco è stata rinvenuta in gran numero, al- lo stadio di larva e di adulto, oltre che su A. artemisiifolia, anche su Helianthus tuberosus* e su Xanthium italicum*.

Osservazioni: specie reperita in 3 delle stazioni di campionamento, in 10 esemplari (Landriano, Perego, Vertaggera).

Diabrotica virgifera virgifera LeConte, 1868

Corotipo: specie di origine Neartica, recentemente segnalata in Italia.

Presenza in Italia: Piemonte, Lombardia, Trentino Alto Adige, Veneto, Friuli Venezia Giulia, Emilia Romagna.

Piante ospiti: è considerata uno dei principali parassiti per le coltivazioni del mais (Zea mays*). Occasionalmente la larva attacca altre Poaceae spontanee o coltivate, mentre gli adulti sono pure rinvenibili su diverse specie di Asteraceae, Cucurbitaceae, Fabaceae (Sassi, 2007).

Osservazioni: specie reperita in 2 delle stazioni di campionamento, in un totale di 2 esemplari (Landriano, Squallera).

Calomicrus circumfusus (Marsham, 1802)

Corotipo: S-Europeo (SEU).

Presenza in Italia: Penisola.

Piante ospiti: Fabaceae (Genista tinctoria*, G. sylvestris, Spartium junceum, Cytisus scoparius*, Ulex).

Osservazioni: specie reperita in un unico esemplare 1'11.6.2013, sui prati magri della stazione di Squallera, su Genista tinctoria*.

Luperus flavipes flavipes (Linné, 1767)

Corotipo: Asiatico-Europeo (ASE).

Presenza in Italia: regioni settentrionali, Toscana.

Piante ospiti: Betulaceae (Alnus, Betula), Salicaceae (Salix aurita), Corylaceae (Corylus avellana*, Ostrya carpinifolia*), Fagaceae (Quercus robur*).

Osservazioni: specie reperita in 2 esemplari il 25.5.2008 a Perego, su Quercus robur.

Luperus leonardii Fogato, 1979

Corotipo: S-Europeo (SEU).

Presenza in Italia: Penisola.

Piante ospiti: Corylaceae (Corylus avellana*), Ulmaceae (Ulmus minor*).

Osservazioni: specie reperita in un unico esemplare 1’11.6.2013 a Squallera, su Corylus avellana.

Luperus longicornis (Fabricius, 1781)

Corotipo: Sibirico-Europeo (SIE).

Presenza in Italia: regioni settentrionali, Appennino settentrionale e centrale.

Piante ospiti: Betulaceae (Betula), Salicaceae (Salix), Corylaceae (Alnus glutinosa*, Corylus avellana*), Fagaceae (Quercus robur*), Rosaceae (Crataegus).

Osservazioni: specie reperita in 3 delle stazioni di campionamento, in un totale di 13 esemplari (Mondonico, Perego, Rovagnate).

Phyllotreta atra (Fabricius, 1775)

Corotipo: Asiatico-Europeo (ASE).

Presenza in Italia: Penisola e Sardegna.

Piante ospiti: Brassicaceae spontanee e coltivate, eccezionalmente Resedaceae e Tropeolaceae. 
Osservazioni: specie reperita in un unico esemplare 1'11.6.2013, a Vertaggera.

\section{Phyllotreta ochripes (Curtis, 1837)}

Corotipo: Sibirico-Europeo (SIE).

Presenza in Italia: regioni settentrionali e centrali, $\mathrm{Pu}-$ glia, Sicilia.

Piante ospiti: Brassicaceae (Alliaria officinalis, A. petiolata*, Sisymbrium, Nasturtium, Diplotaxis muralis*, Cardamine amara, C. pentaphyllos, Rorippa amphibia, Sinapis arvensis*, Brassica napus*). Gli adulti trascorrono l'inverno nascosti tra le foglie secche ai piedi delle piante ospiti (Doguet, 1994).

Osservazioni: specie reperita in un unico esemplare il 27.4.2014 a Squallera, su prato da foraggio.

Phyllotreta punctulata (Marsham, 1802)

Corotipo: Europeo (EUR).

Presenza in Italia: tutte le regioni.

Piante ospiti: Brassicaceae spontanee e coltivate (Brassica, Sinapis, Erysimum, Diplotaxis, Alliaria, Armoracia, Lepidium), Resedaceae (Reseda).

Osservazioni: specie reperita in un unico esemplare il 2.4.2013 a Perego, sui prati aridi.

\section{Phyllotreta vittula (Redtenbacher,1849)}

Corotipo: Asiatico-Europeo (ASE).

Presenza in Italia: Penisola.

Piante ospiti: abitualmente su Brassicaceae, Poaceae e Cyperaceae, eccezionalmente su Asteraceae e Chenopodiaceae. Specie monovoltina. L'adulto trascorre l'inverno in diversi rifugi, al suolo oppure nascosto sotto la corteccia degli alberi (Doguet, 1994).

Osservazioni: specie reperita in 8 delle stazioni di campionamento, in un totale di 257 esemplari (Cà del Soldato, Calendone, Landriano, Perego, Rovagnate, Squallera, Vertaggera).

\section{Aphthona herbigrada (Curtis, 1837)}

Corotipo: Europeo-Mediterraneo (EUM).

Presenza in Italia: tutte le regioni.

Piante ospiti: Cistaceae del genere Helianthemum.

Osservazioni: specie reperita in 3 delle stazioni di campionamento, in un totale di 48 esemplari (Perego, Rovagnate, Vertaggera).

\section{Aphthona lutescens (Gyllenhal, 1808)}

Corotipo: Centroasiatico-Europeo (CAE).

Presenza in Italia: Penisola e Sardegna.

Piante ospiti: Lythraceae (Lythrum salicaria*), Rosaceae (Filipendula ulmaria*) e Lamiaceae (Mentha acquatica*).

Osservazioni: specie reperita in un unico esemplare il 10.8.2012 nella stazione di Calendone.

\section{Aphthona ovata Foudras, 1860}

Corotipo: Europeo (EUR).

Presenza in Italia: regioni settentrionali, Toscana, Lazio, Calabria, Sicilia.

Piante ospiti: Euphorbiaceae (Euphorbia cyparissias*, Euphorbia).
Osservazioni: specie reperita solo sui prati magri di Perego per un totale di 3 esemplari.

\section{Aphthona sicelidis Weise, 1888}

Corotipo: Mediterraneo (MED).

La specie, descritta di Sicilia, è citata anche di Francia meridionale, Liguria, Umbria, Toscana, Calabria (Heikertinger, 1912; 1944; Porta, 1934; Biondi, 1990; Doguet, 1994). Il Parco del Curone, primo dato lombardo, rappresenta il limite nord dell'areale (Fig. 4). Ciò è indice del carattere mediterraneo del biotopo dei prati magri. Nelle collezioni del Museo Civico di Storia Naturale di Milano sono conservati anche esemplari lombardi dell'Oltrepò pavese (Lago di Trebecco) e di Puglia.

Habitat: la specie è stata rinvenuta su praterie terrazzate semiaride nelle stazioni di Perego, Rovagnate e Vertaggera da aprile ad ottobre, per un totale di 27 esemplari.

Piante ospiti: Euphorbia (Heikertinger, 1944).

Aphthona sicelidis, descritta da Weise come specie distinta, è stata successivamente trattata da Heikertinger come sottospecie di A. bonvouloiri All., e come tale è rimasta fino a tempi recenti (1998), quando Konstantinov, dopo averne fissato il Lectotypus su una + conservata presso il Museo di Storia Naturale di Budapest, ha ritenuto opportuno riabilitarla.

Incomprensibilmente Konstantinov, senza aver esaminato alcun $\widehat{\partial} \mathrm{di} A$. sicelidis, ne limita l'areale alla sola Sicilia, mentre cita genericamente di "Italia" A. bonvouloiri. In realtà $A$. bonvouloiri è diffusa solo nel Mediterraneo orientale, mentre i numerosi individui dell'Italia peninsulare che ho potuto esaminare non differiscono sostanzialmente da quelli siciliani.

Come aveva osservato Heikertinger, che nel 1912 ne descrisse l'aberrazione obscuripes, in A. sicelidis le zampe hanno una colorazione molto variabile; al contrario in A. bonvouloiri sono costantemente chiare (ad eccezione dei femori posteriori che possono essere più o meno oscurati), in quanto la forma con zampe picee, che le era stata attribuita, è stata recentemente descritta da Fritzlar (2001) come Aphthona bergeali.

A. sicelidis e A. bonvouloiri possono essere separate soprattutto in base ai seguenti caratteri

1. edeago in visione ventrale non o poco percettibilmente ristretto a metà della sua lunghezza, in visione laterale sensibilmente sinuato distalmente (Fig. 5a; v. anche Konstantinov, 1998: fig. 504; Fritzlar, 2001: tav.2, Fig. 3a-c). Parte basale della spermateca sensibilmente ristretta verso il ductus (Fig. 6i, 1; v. anche Konstantinov, 1998: fig. 206; Fritzlar, 2001: tav. 1, Fig. 3f). Rami apicali dello spiculum sottili, debolmente allargati distalmente (Fig. 6d; v. anche Konstantinov, 1998: fig. 405; Fritzlar, 2001: tav. 2, Fig. 3d) .................... A. bonvouloiri Allard

1'. edeago in visione ventrale debolmente ma visibilmente ristretto circa a metà della sua lunghezza, in visione laterale non o molto debolmente sinuato distalmente (Fig. 5b; v. anche Doguet, 1994: fig. 73a; Fritzlar, 2001: tav.2, Fig. 4a-c). Spermateca generalmente più piccola. Parte basale della spermateca subcilindrica, in genere leggermente reniforme (Fig. 6e-h; v. anche Konstantinov, 1998: fig. 279; Fritzlar, 2001: tav.1, Figg. 4f', 4f'). Rami apicali dello spiculum più o meno dilatati (Fig. 6a-c; v. anche Konstantinov, 1998: fig. 477; Fritzlar, 2001: tav. 2, Fig. 4d) ....................... A. sicelidis Weise 

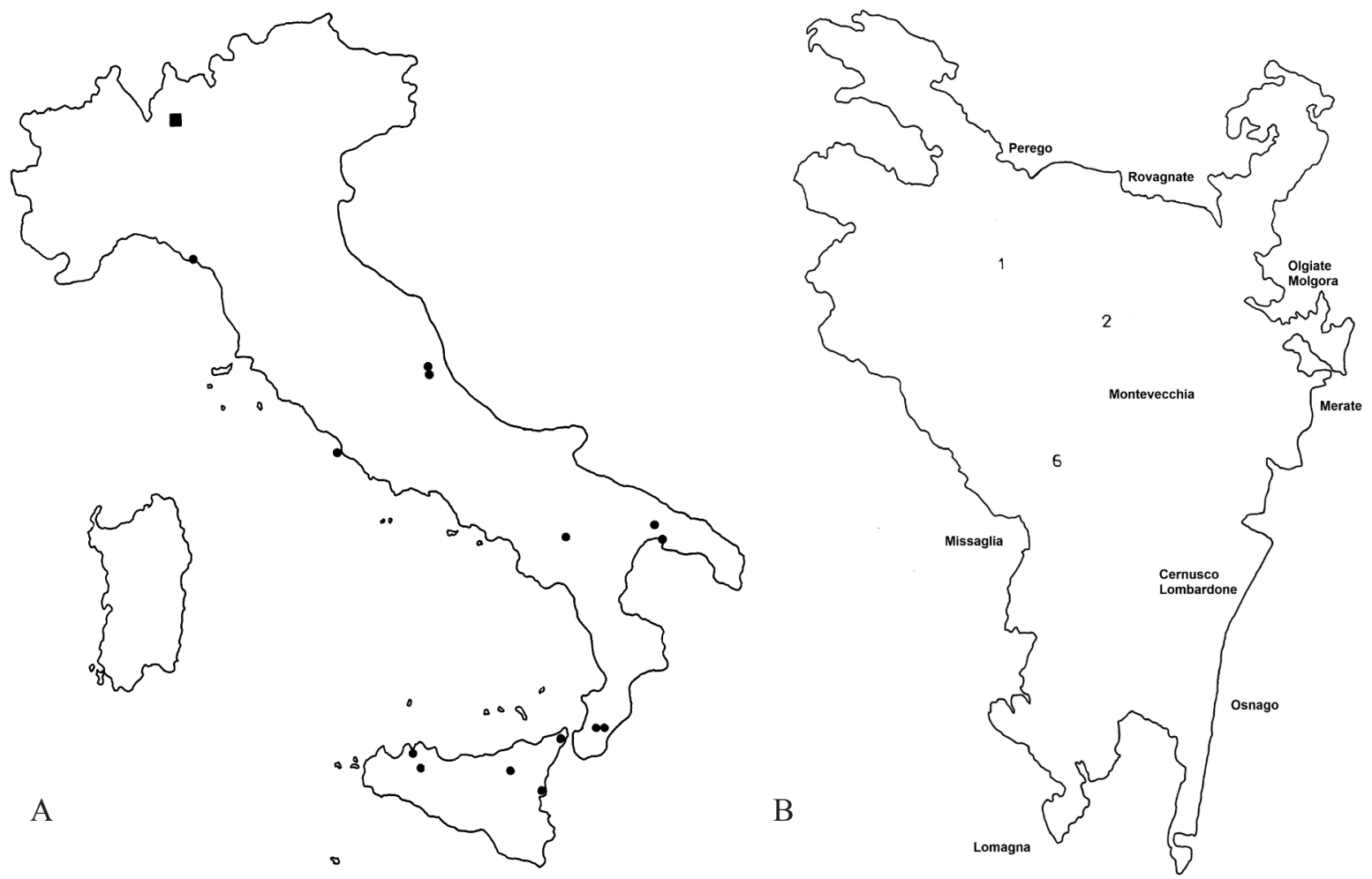

Fig. 4 - Aphthona sicelidis. A) distribuzione sul territorio italiano (CKmap, 2006) con nuova indicazione per il Parco (quadratino). B) distribuzione nel Parco del Curone.
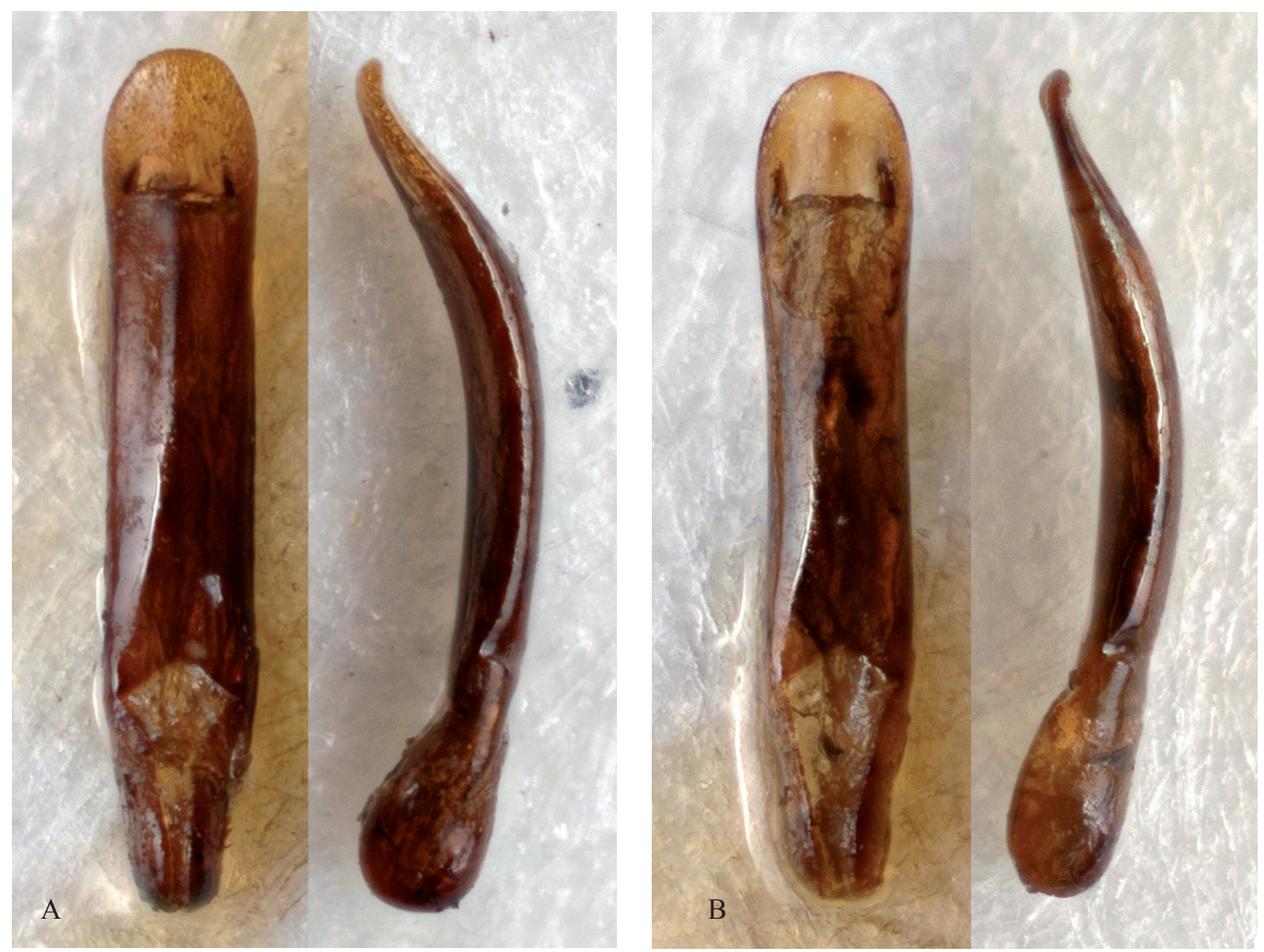

Fig. 5 - Lobo mediano dell'edeago, in visione ventrale e laterale. A) Aphthona bonvouloiri (lunghezza: 0,82 mm). B) Aphthona sicelidis (lunghezza: 0,73 mm). Località degli esemplari fotografati: bonvouloiri: Dinar (Turchia), sicelidis: Parco del Curone (Perego). (Foto V. Fogato). 

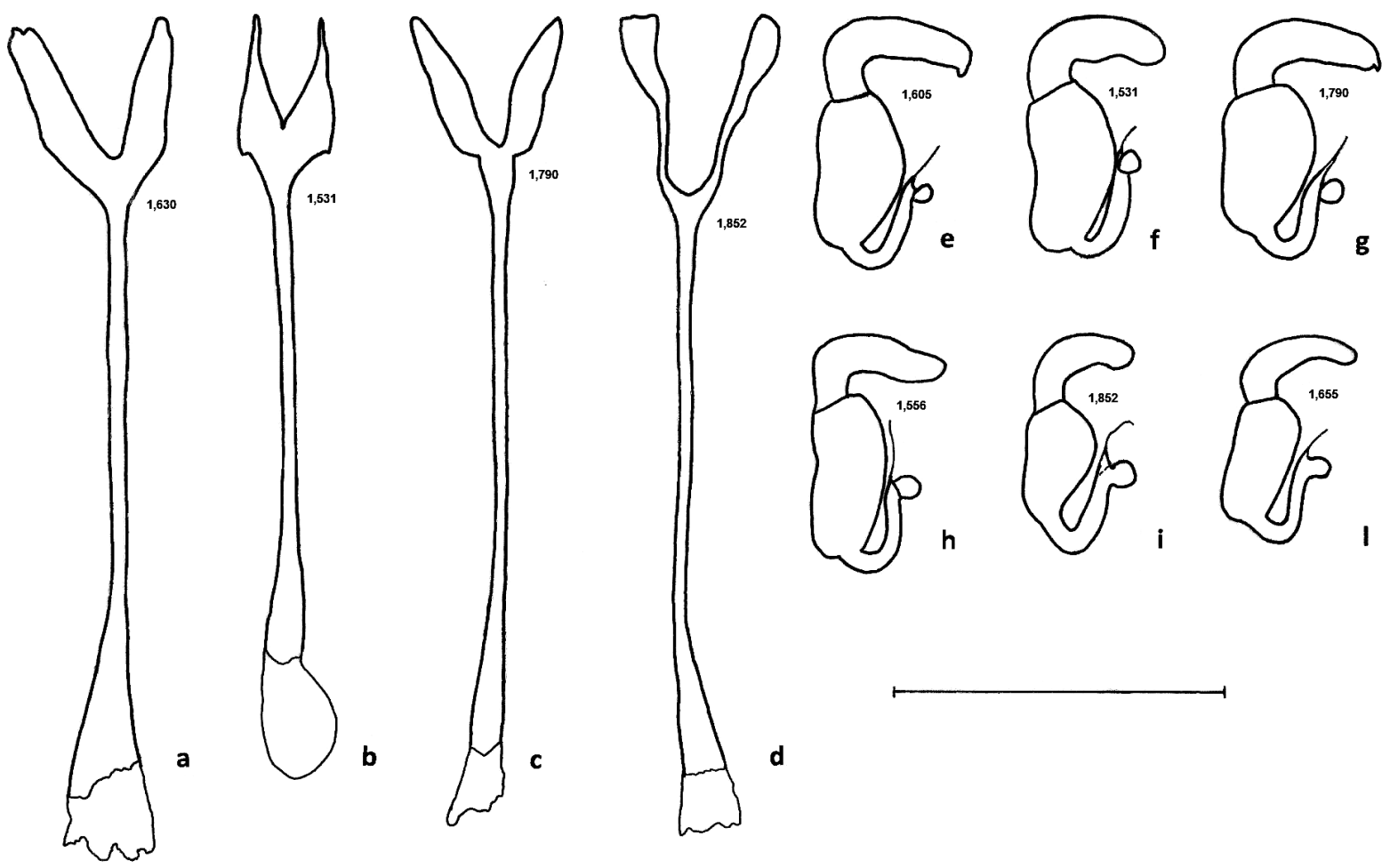

Fig. 6 - a-d) spicula. a-b) Aphthona sicelidis, Perego (Parco del Curone); c) Aphthona sicelidis, Francavilla di Sicilia (Messina); d) Aphthona bonvouloiri, Sud di Adana (Cilicia). e-l) spermateca. e-f) Aphthona sicelidis, Perego (Parco del Curone); g) Aphthona sicelidis, Francavilla di Sicilia (Messina); h) Aphthona sicelidis, Todi (Umbria); i) Aphthona bonvouloiri, Sud di Adana (Cilicia); 1) Aphthona bonvouloiri, Tel Aviv. (Scala 0,3 mm). I numeri indicano la lunghezza elitrale (in $\mathrm{mm}$ ) degli esemplari da cui sono stati ricavati $\mathrm{i}$ disegni.

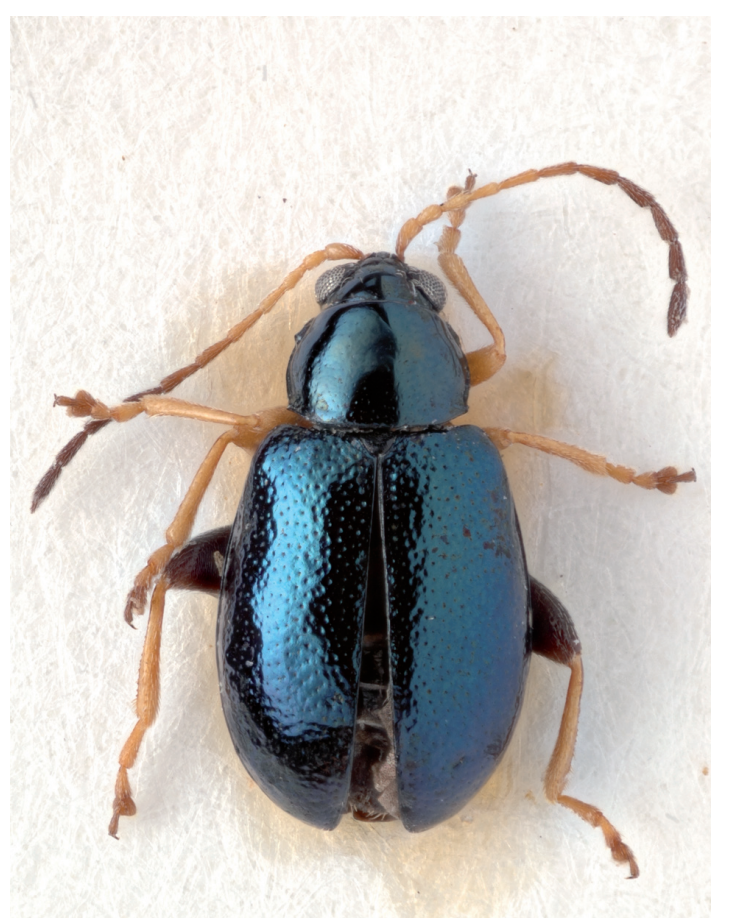

A

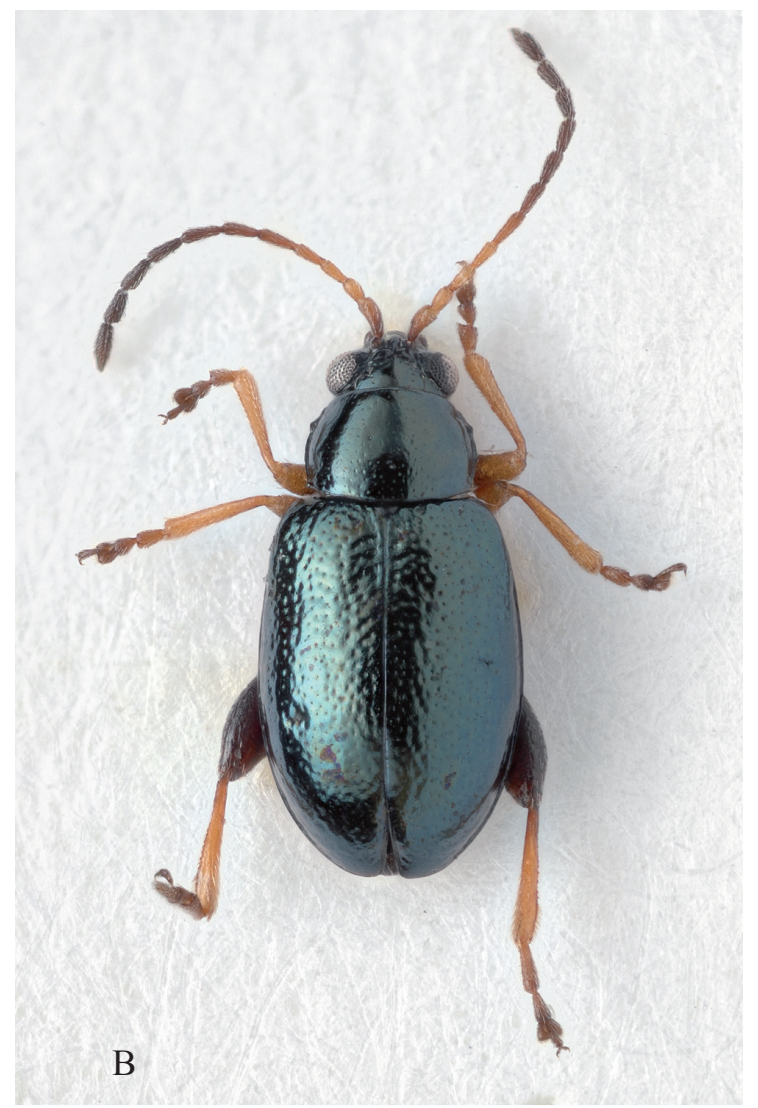

Fig. 7 - Differenze cromatiche. A) Aphthona sicelidis (Sicilia) (lunghezza: 2,14 mm); B) Aphthona sicelidis (Parco del Curone) (lunghezza: $1,98 \mathrm{~mm}$ ). (Foto V. Fogato). 
A. sicelidis, come giustamente riteneva Weise (1888), è vicina anche ad $A$. venustula, ma se ne distingue agevolmente per la punteggiatura elitrale più forte e per i riflessi metallici molto più accentuati, che in genere sono dichiaratamente azzurri, mentre la forma del Parco del Curone è tendenzialmente verde (Fig. 7).

\section{Materiale esaminato}

\section{Aphthona sicelidis}

Numerosi esemplari delle seguenti località: Sicilia: Bosco della Ficuzza (MMi, MBa), Messina (MBa), Monti Peloritani (Messina) (MMi), Siracusa (MMi), Palermo (MBa), Francavilla di Sicilia, fiume Alcantara (MMi); Calabria: Gerace (MBa); Puglia: Martina (Taranto), Bosco Pianelle (MMi), Riserva Naturale Le Cesine (Lecce) (CMt), Terra d'Otranto, Castro (MMi); Toscana: Isola d'Elba, Sant'Ilario in Campo (MMi), Pisa/Arno (MBa); Umbria: Todi (MMi); Liguria: Chiavari/Leivi (MMi), San Lorenzo di Casanova (Genova) (MMi), Monte Portofino (Genova) (MMi), Cogoleto-Rio Lerca (Savona) (MMi), Monte Sciguello (Savona) (MMi) Levanto (La Spezia) (MMi); Lombardia: Lago di Trebecco (MMi), Parco del Curone (CFa). Raccolte nel Parco del Curone: Perego, leg. L. Farina: $1 \sigma^{\lambda}$ 4 우우 1.8.2012; Montevecchia, Vertaggera, leg. L. Farina: $1 \hat{\delta} 1$ ㅇ 17.7.2012; Rovagnate, Monte, leg. L. Farina: $1 \delta^{\lambda}$ 1.8.2012; Perego, leg. L. Farina: 19 5.9.2012; Perego, leg.

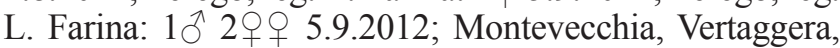
leg. L. Farina: $10^{\lambda}$ 20.9.2012; Perego, leg. L. Farina: $4{ }^{\lambda} \partial^{\lambda}$ 4 우 24.10.2012; Perego, leg. L. Farina: $1 \delta^{\widehat{o}} 1$ ㅇ 2.4.2013;

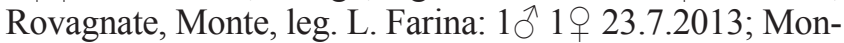
tevecchia, Vertaggera, leg. L. Farina: $1 \delta$ 23.8.2013.

In base al materiale che è stato possibile esaminare Aphthona sicelidis è di fatto un endemita italiano; l'unico esemplare $\left({ }^{\Uparrow}\right)$ di provenienza francese (Savoie) è problematico, perché il suo edeago (Doguet, 1994: fig. 73b) è identico a quello di $A$. bonvouloiri; non è da escludere un errore di etichettatura.

\section{Aphthona bonvouloiri}

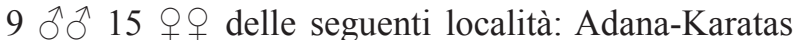
(Cilicia) (MMi), Amanusgebiet/Jaribaschi (MBa), Belemedik (Cilicic Taurus) (MBa), Dinar (Turchia) (MMi) Içel ad Mersin (Turchia mer.) (MMi, coll. Bergeal), Haifa (Syrien) (MBa), Tel-Aviv (Palestina) (MMi), Cairo (Ägypten), Insel Rhodos.

Abbreviazioni utilizzate: $\mathrm{MMi}=$ Museo Civico di Storia Naturale di Milano, $\mathrm{MBa}=$ Naturhistorisches Museum Basel, $\mathrm{CMt}=$ collezione Montemurro, $\mathrm{CFa}=$ Collezione dell'autrice.

\section{Aphthona venustula Kutschera, 1861 \\ Corotipo: Europeo (EUR). \\ Presenza in Italia: Penisola. \\ Piante ospiti: Euphorbiaceae (Euphorbia cyparissias*, Euphorbia).}

Osservazioni: specie reperita in un unico esemplare il 13.4.2013 a Mondonico, su prato umido.

\section{Longitarsus (Longitarsus) apicalis (Beck,1817)}

Corotipo: Sibirico-Europeo (SIE).
Presenza in Italia: regioni settentrionali, Toscana e Campania.

Piante ospiti: Asteraceae (Cirsium, Carduus, Leontodon autumnalis*), Apiaceae (Chaerophyllum hirsutum), Lamiaceae (Lotus corniculatus*).

Osservazioni: la specie è stata rinvenuta sui prati umidi, in 2 delle stazioni di campionamento, in un totale di 3 esemplari (Cà del Soldato, Mondonico).

Longitarsus (Longitarsus) codinai Madar \& Madar, 1965 Corotipo: W-Mediterraneo (WME).

Presenza in Italia: Liguria, Lombardia, Emilia Romagna, Toscana, Marche, Abruzzo, Calabria, Sicilia e Sardegna.

Piante ospiti: Convolvulaceae (Convolvulus).

Osservazioni: specie reperita in un unico esemplare il 5.9.2012 sui prati magri di Perego.

Longitarsus (Longitarsus) helvolus Kutschera, 1864 Corotipo: Centroeuropeo (CEU).

Presenza in Italia: Piemonte, Liguria, Lombardia, Trentino Alto Adige, Veneto, Friuli Venezia Giulia e Calabria.

Piante ospiti: Lamiaceae (Teucrium).

Osservazioni: la specie è stata reperita sulle praterie xerotermiche in 2 delle stazioni di campionamento, in un totale di 4 esemplari (Perego, Rovagnate).

Longitarsus (Longitarsus) lewisii (Baly, 1874)

Corotipo: Asiatico-Europeo (ASE).

Presenza in Italia: Valle d'Aosta, Piemonte, Lombardia, Trentino Alto Adige, Veneto, Friuli Venezia Giulia, Emilia Romagna.

Piante ospiti: Plantaginaceae (Plantago), Lamiaceae (Mentha arvensis*). Specie bivoltina (Leonardi in Leonardi \& Doguet, 1990).

Osservazioni: specie reperita in 3 delle stazioni di campionamento, in un totale di 5 esemplari (Landriano, Perego, Vertaggera).

Longitarsus (Longitarsus) luridus luridus (Scopoli, 1763) Corotipo: Paleartico (PAL).

Presenza in Italia: tutte le regioni.

Piante ospiti: Ranunculaceae, Boraginaceae, Lamiaceae, Plantaginaceae, Dipsacaceae, Scrophulariaceae. Specie bivoltina (Heikertinger, 1926).

Osservazioni: specie reperita in 7 delle stazioni di campionamento, in un totale di 100 esemplari (Cà del Soldato, Calendone, Mondonico, Perego, Rovagnate, Squallera, Vertaggera).

Longitarsus (Longitarsus) melanocephalus (De Geer, 1775) Corotipo: Asiatico-Europeo (ASE).

Presenza in Italia: Penisola, Sicilia e Sardegna.

Piante ospiti: Plantaginaceae (Plantago). L'adulto trascorre l'inverno nascosto al suolo tra i detriti.

Osservazioni: specie reperita in 6 delle stazioni di campionamento, in un totale di 27 esemplari (Cà del Soldato, Mondonico, Perego, Squallera, Valle S. Croce, Vertaggera).

Longitarsus (Longitarsus) obliteratus (Rosenhauer, 1847) Corotipo: Europeo (EUR). 
Presenza in Italia: Penisola.

Piante ospiti: Lamiaceae (Salvia pratensis*, S. nemorosa, $S$. verticillata*, Thymus serpyllum, $T$. vulgaris, $S a-$ tureja montana, Origanum vulgare*, Clinopodium vulgare*, Prunella, Melissa officinalis*, Stachys recta*).

Osservazioni: la specie è stata reperita su Origanum vulgare in 2 stazioni di campionamento, in un totale di 20 esemplari (Perego, Rovagnate).

Longitarsus (Longitarsus) pellucidus (Foudras, 1860) Corotipo: Centroasiatico-Europeo-Mediterraneo (CEM). Presenza in Italia: tutte le regioni.

Piante ospiti: Convolvulaceae (Convolvulus arvensis*, più raramente su Calystegia sepium*). L'adulto trascorre l'inverno nascosto tra le cortecce degli alberi.

Osservazioni: la specie è stata reperita in 4 stazioni di campionamento, in un totale di 32 esemplari (Perego, Rovagnate, Valle S. Croce, Vertaggera).

Longitarsus (Longitarsus) pratensis (Panzer, 1794)

Corotipo: W-Paleartico (WPA).

Presenza in Italia: tutte le regioni.

Piante ospiti: Plantaginaceae (Plantago major*, $P$. lanceolata*). Specie probabilmente bivoltina (Heikertinger, 1926 e Leonardi in Leonardi \& Doguet, 1990).

Osservazioni: la specie è stata reperita in 8 stazioni di campionamento, in un totale di 225 esemplari (Cà del Soldato, Landriano, Mondonico, Perego, Rovagnate, Squallera, Valle S. Croce, Vertaggera).

Longitarsus (Longitarsus) salviae Gruev, 1975

Corotipo: Centroeuropeo (CEU).

Presenza in Italia: Lombardia, Veneto, Friuli Venezia Giulia, Puglia, Calabria.

Piante ospiti: Lamiaceae (Salvia pratensis*, Salvia).

Osservazioni: la specie è stata reperita in 2 stazioni di campionamento, in un totale di 42 esemplari (Perego, Rovagnate).

Longitarsus (Longitarsus) succineus (Foudras, 1860)

Corotipo: Paleartico (PAL).

Presenza in Italia: tutte le regioni.

Piante ospiti: Convolvulaceae, Lamiaceae, Plantaginaceae, Boraginaceae, Asteraceae.

Osservazioni: la specie è stata reperita in 6 stazioni di campionamento, in un totale di 63 esemplari (Landriano, Mondonico, Perego, Rovagnate, Squallera, Vertaggera).

\section{Altica brevicollis brevicollis Foudras, 1861}

Corotipo: Turanico-Europeo (TUE).

Presenza in Italia: Penisola.

Piante ospiti: Corylaceae (Corylus avellana). Probabilmente la specie è bivoltina (Thérond, 1976).

Osservazioni: specie reperita in un'unica stazione, in un totale di 3 esemplari, a Cà del Soldato, su Corylus avellana.

Altica oleracea oleracea (Linné, 1758)

Corotipo: Asiatico-Europeo (ASE).

Presenza in Italia: tutte le regioni.

Piante ospiti: specie polifaga: abitualmente Ericace- ae, Polygonaceae, Onagraceae; occasionalmente Vitaceae, Rosaceae, Scrophulariaceae, Corylaceae, Fagaceae e Lythraceae. La specie presenta probabilmente più generazioni l'anno.

Osservazioni: la specie è stata reperita in 6 stazioni di campionamento, in un totale di 44 esemplari (Landriano, Mondonico, Perego, Rovagnate, Squallera, Vertaggera).

\section{Lythraria salicariae (Paikull, 1800)}

Corotipo: Asiatico-Europeo (ASE).

Presenza in Italia: regioni settentrionali e centrali, Campania, Basilicata, Calabria.

Piante ospiti: Primulaceae (Lysimachia vulgaris*, $L$. punctata), Lythraceae (Lythrum salicaria*, L. thesioides). L'adulto trascorre l'inverno nascosto tra i detriti (Galibert, 1932).

Osservazioni: specie reperita sui prati umidi, in un totale di 15 esemplari, a Mondonico su Lythrum salicaria e a Cà del Soldato su Lysimachia vulgaris.

Crepidodera aurata (Marsham, 1802)

Corotipo: Asiatico-Europeo (ASE).

Presenza in Italia: tutte le regioni.

Piante ospiti: Salicaceae (Populus tremula*, P. alba*, P. nigra*, Salix).

Osservazioni: la specie è stata reperita in 7 stazioni di campionamento, in un totale di 171 esemplari (Cà del Soldato, Calendone, Mondonico, Perego, Rovagnate, Squallera, Vertaggera).

Crepidodera fulvicornis (Fabricius, 1792)

Corotipo: Asiatico-Europeo (ASE).

Presenza in Italia: Italia settentrionale e centrale. Prima segnalazione della specie per la regione Lombardia.

Piante ospiti: Doguet (1994) segnala la specie su Salix (S. aurita, S. repens, S. caprea*, S. elaeagnos) e su Populus tremula*. Nel Parco C. fulvicornis è stata raccolta esclusivamente su Salix cinerea.

Osservazioni: specie reperita in un totale di 9 esemplari su Salix cinerea, lungo lo stagno di Calendone (Merate).

In letteratura, la specie (Fig. 8) risulta genericamente nota dell'Italia settentrionale (Porta, 1934) e di alcune regioni centrali. Le citazioni riguardano più in particolare: Veneto, Friuli Venezia Giulia (Müller, 1953), Toscana (Porta, 1934; Bordoni, 1972), Umbria, Marche (Biondi, 1994), Abruzzo, Molise (Biondi et al., 1992).

\section{Materiale esaminato}

Alcuni esemplari delle seguenti località in Lombardia: Cusago (Milano, dintorni) (MMi); Lago di Ganna (Varese) (CZo). Raccolte nel Parco del Curone (CFa): Calendone, leg. L. Farina: 1 ㅇ 10.8.2012; 2 ổ 6 우 14.6.2014.

Abbreviazioni utilizzate: $\mathrm{MMi}=$ Museo Civico di Storia Naturale di Milano, $\mathrm{CZo}=$ collezione Zoia, $\mathrm{CFa}=$ Collezione dell'autrice.

Podagrica malvae malvae (Illiger, 1807)

Corotipo: Centroeuropeo (CEU).

Presenza in Italia: tutte le regioni. 


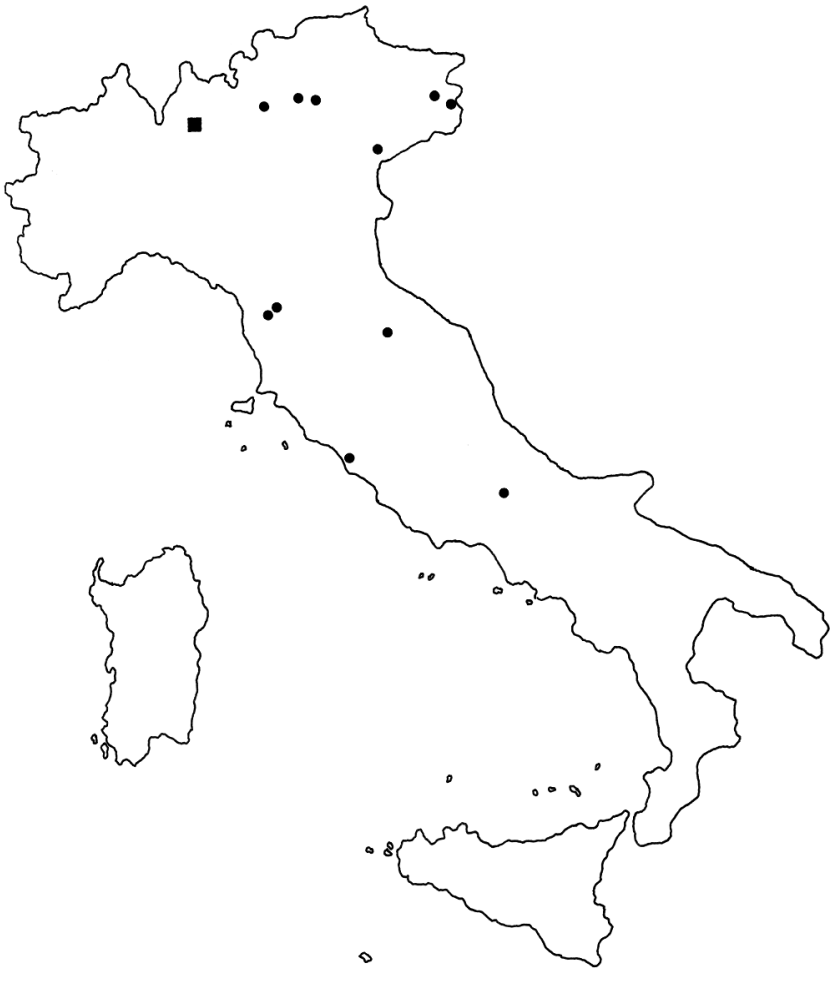

A

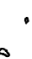

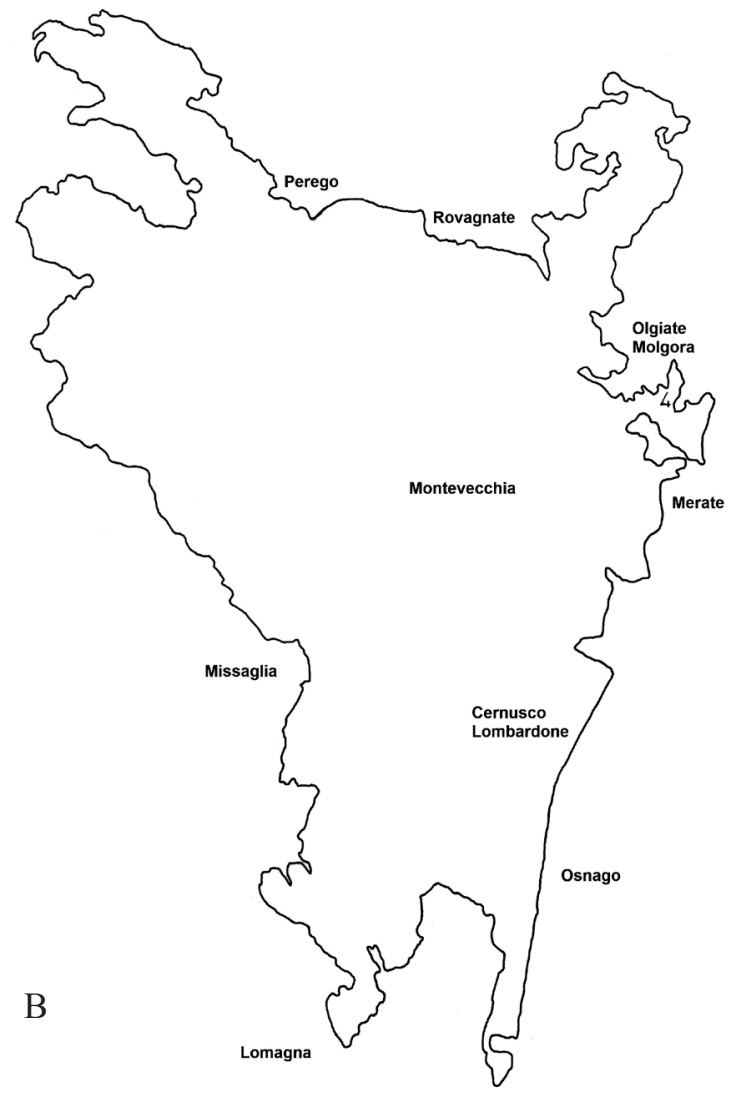

Fig. 8 - Crepidodera fulvicornis. A) distribuzione sul territorio italiano (CKmap, 2006) con nuova indicazione per il Parco (quadratino). B) distribuzione nel Parco.

Piante ospiti: prevalentemente Malvaceae (Malva, Alcea, Hibiscus, Lavatera), ma segnalata anche su Cistaceae, Asteraceae, Lamiaceae, Boraginaceae sia spontanee che coltivate. La specie può anche nutrirsi, da adulto, di foglie di Urtica.

Osservazioni: la specie è stata reperita in 2 stazioni di campionamento, in un totale di 2 esemplari (Mondonico, Perego).

Chaetocnema (Tlanoma) concinna (Marsham, 1802)

Corotipo: Asiatico-Europeo (ASE).

Presenza in Italia: Penisola e Sicilia. Presenza da confermare in Sardegna.

Piante ospiti: Polygonaceae (Polygonum, Rumex, Rheum raphonticum, Fagopyrum tataricum), Chenopodiaceae (Atriplex, Beta vulgaris*).

Osservazioni: la specie è stata reperita in 4 stazioni di campionamento, in un totale di 12 esemplari (Mondonico, Perego, Squallera, Vertaggera).

\section{Chaetocnema (Tlanoma) conducta (Motschulski, 1838)}

Corotipo: Turanico-Europeo-Mediterraneo (TEM).

Presenza in Italia: Penisola e Sicilia. Presenza da confermare per la Sardegna.

Piante ospiti: Cyperaceae (Eleocharis palustris, $\mathrm{Ca}$ rex, Bolboschoenus maritimus), Juancaceae (Juncus), Poaceae (Agrostis alba). La specie attacca alcune piante coltivate: rabarbaro (Rheum rhaponticum) e grano saraceno (Fagopyrum esculentum*).
Osservazioni: la specie è stata reperita in tutte le stazioni di campionamento, in un totale di 88 esemplari.

Chaetocnema (Chaetocnema) hortensis (Geoffroy, 1785)

Corotipo: Indiano-Mediterraneo (INM). Secondariamente Afrotropicale.

Presenza in Italia: Penisola e Sicilia.

Piante ospiti: Cyperaceae, Poaceae (Sesleria caerulea*, Arrhenatherum elatius*, Avena, Triticum, Hordeum vulgare*).

Osservazioni: la specie è stata reperita in tutte le stazioni di campionamento, in un totale di 119 esemplari.

Chaetocnema (Tlanoma) picipes Stephens, 1831

Corotipo: Asiatico-Europeo (ASE).

Presenza in Italia: Penisola.

Piante ospiti: Polygonaceae (Polygonum persicaria*, P. aviculare*, Brassica rapa).

Osservazioni: la specie è stata reperita in 3 stazioni di campionamento, in un totale di 7 esemplari (Calendone, Perego, Rovagnate).

Chaetocnema (Tlanoma) tibialis (Illiger, 1807)

Corotipo: W-Paleartico (WPA).

Presenza in Italia: Penisola, Sardegna e Isola d'Elba.

Piante ospiti: Chenopodiaceae. La specie presenta una sola generazione annuale. Allo stadio di immagine può compiere danni alle foglie delle giovani piante di barbabietola. La specie è stata anche segnalata su altre 
piante coltivate: Spinacea oleracea e amaranti ornamentali.

Osservazioni: la specie è stata reperita in 2 stazioni di campionamento, in un totale di 8 esemplari (Mondonico, Vertaggera).

\section{Sphaeroderma rubidum (Graëlls, 1858)}

Corotipo: Europeo-Mediterraneo (EUM).

Presenza in Italia: tutte le regioni.

Piante ospiti: Asteraceae (Centaurea, Carduus, Cirsium).

Osservazioni: la specie è stata reperita in 6 stazioni di campionamento, in un totale di 96 esemplari (Mondonico, Perego, Rovagnate, Squallera, Valle S. Croce, Vertaggera).

Dibolia (Eudibolia) femoralis Redtenbacher, 1849

Corotipo: S-Europeo (SEU).

Presenza in Italia: Italia settentrionale e centrale. Prima segnalazione della specie per la regione Lombardia.

Piante ospiti: Salvia.

Osservazioni: specie reperita in un totale di 2 esemplari sui prati magri di Perego.

In letteratura la specie (Fig. 9) risulta nota genericamente dell'Italia settentrionale e centrale (Luigioni, 1929; Porta, 1934). Le citazioni riguardano più in particolare: Campania (Porta, 1934), Puglia (Holdhaus K., 1911; Porta, 1934), Piemonte (Bertolini, 1886; Della Beffa, 1911), Trentino-Alto Adige (Bertolini, 1886; Halbherr, 1898), Veneto (Müller, 1953; Zangheri, 1969), Friuli Venezia Giulia (Müller, 1953), Toscana (Bertolini, 1886).

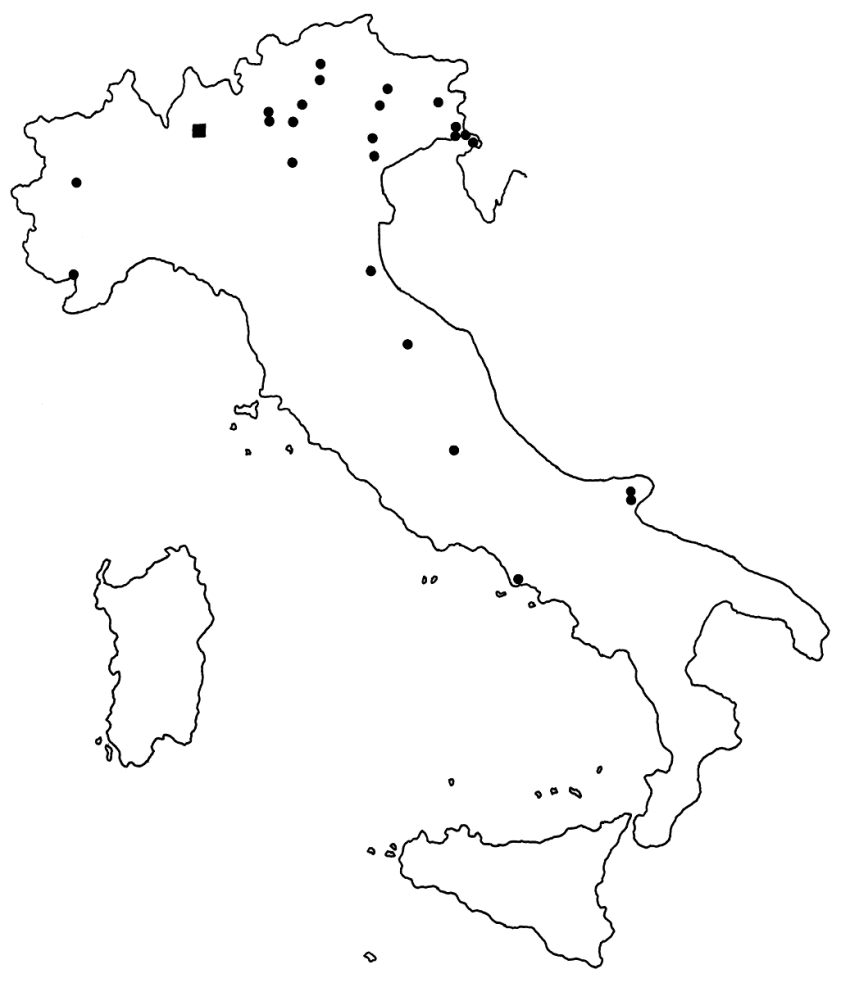

A

\section{Materiale esaminato}

Diversi esemplari in Lombardia provenienti da: Gaggiolo, Varese; Travaco, Pavia, confluenza Po-Ticino; Calolzio (MMi). Raccolte nel Parco del Curone $(\mathrm{CFa})$ : Perego, leg. L. Farina: 19 1.8.2012; 1 ๙ 16.8.2013.

Abbreviazioni utilizzate: $\mathrm{MMi}=$ Museo Civico di Storia $\mathrm{CFa}=$ Collezione dell'autrice.

Dibolia (Dibolia) rugulosa Redtenbacher, 1849

Corotipo: Europeo (EUR).

Presenza in Italia: Valle d'Aosta, Piemonte, Lombardia, trentino Alto Adige, Friuli Venezia Giulia, Toscana, Lazio e Abruzzo.

Piante ospiti: Lamiaceae (principalmente Stachys recta*, segnalata anche su S. annua* e S. sylvatica*).

Osservazioni: la specie è stata reperita su praterie xerotermiche in 2 stazioni di campionamento, in un totale di 6 esemplari (Perego, Rovagnate).

\section{Psylliodes cuprea (Koch, 1803)}

Corotipo: Centroasiatico-Europeo-Mediterraneo (CEM)

Presenza in Italia: tutte le regioni.

Piante ospiti: Brassicaceae. Come diverse altre specie di Alticini, gli adulti si disperdono in estate sugli alberi e sugli arbusti.

Osservazioni: la specie è stata reperita in 2 esemplari a Rovagnate, lungo il sentiero che conduce ai prati magri il 6.11.2013. Gli esemplari rinvenuti sono entrambi femmine.

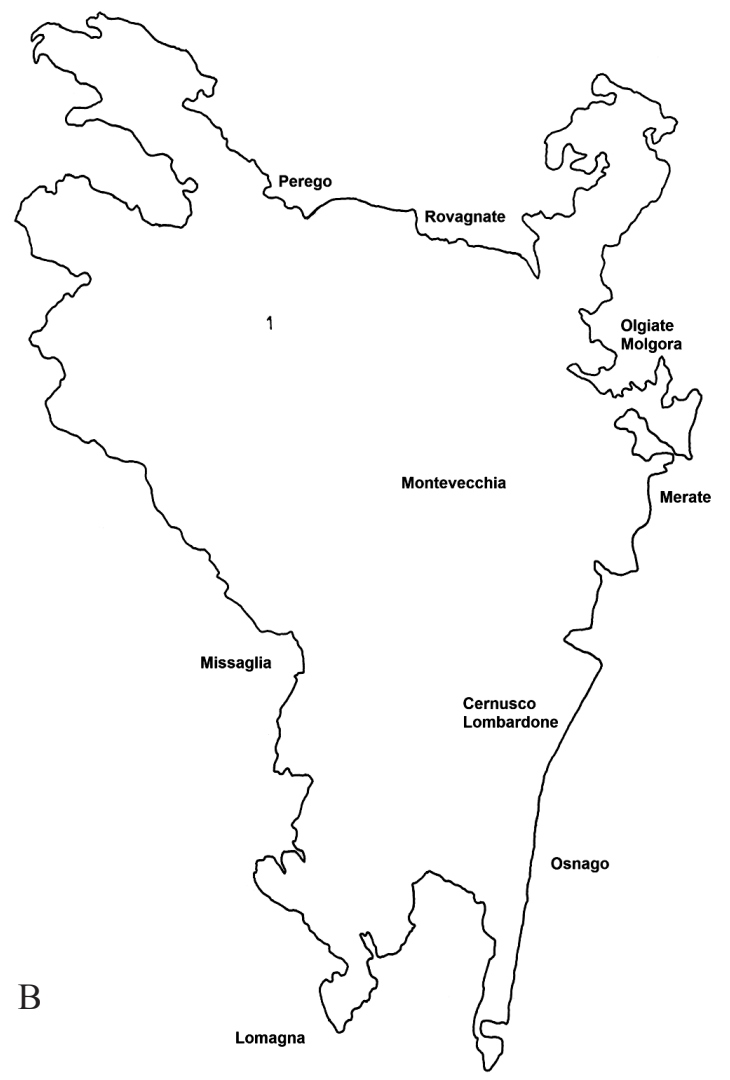

Fig. 9 - Dibolia femoralis. A) distribuzione sul territorio italiano (CKmap, 2006) con nuova indicazione per il Parco (quadratino). B) distribuzione nel Parco. 
Psylliodes gibbosa Allard, 1860

Corotipo: Mediterraneo (MED).

Presenza in Italia: tutte le regioni.

Piante ospiti: Poaceae. Segnalata anche su Sinapis pubescens (Brassicaceae).

Osservazioni: la specie è stata reperita su praterie xerotermiche in 2 stazioni di campionamento, in un totale di 2 esemplari (Mondonico, Vertaggera).

Psylliodes napi (Fabricius, 1792)

Corotipo: Europeo (EUR).

Presenza in Italia: tutte le regioni.

Piante ospiti: Brassicaceae (Alliaria officinalis, $\mathrm{Na}$ sturtium officinale*, Cardamine amara, Brassica, Sinapis, Barbarea vulgaris*).

Osservazioni: la specie è stata reperita su praterie xerotermiche in 3 stazioni di campionamento, in un totale di 4 esemplari (Mondonico, Rovagnate, Vertaggera).

\section{Labidostomis (Labidostomis) humeralis (Schneider,} 1792)

Corotipo: Europeo (EUR).

Presenza in Italia: Veneto, Trentino-Alto Adige, Lombardia, Piemonte, Valle D'Aosta, Liguria, Emilia-Romagna, Toscana, Puglia.

Piante ospiti: la specie è polifaga legata a diversi generi (piante erbacee, alberi e arbusti) delle seguenti famiglie: Betulaceae, Fabaceae, Fagaceae, Polygonaceae, Rosaceae, Salicaceae.

Osservazioni: la specie è stata reperita in un'unica stazione di campionamento, Rovagnate, in un totale di 8 esemplari.

\section{Labidostomis (Labidostomis) longimana (Linné, 1761)}

Corotipo: Turanico-Europeo (TUE).

Presenza in Italia: Penisola. Presenza da confermare per la Sicilia.

Piante ospiti: Fabaceae (Lotus, Trifolium, Dorycnium). Secondo Balachowski (1963) anche su Brassicaceae selvatiche e cereali coltivati.

Osservazioni: la specie è stata rinvenuta su prato umido in 2 stazioni di campionamento in un totale di 2 esemplari (Calendone, Mondonico).

Labidostomis (Labidostomis) lucida (Germar, 1824)

Corotipo: Centroasiatico-Europeo (CAE).

Presenza in Italia: regioni settentrionali e Abruzzo (Maiella, Gran Sasso).

Piante ospiti: Fabaceae (Lotus, Trifolium, Dorycnium), Polygonaceae (Rumex).

Osservazioni: la specie è stata rinvenuta in un unico esemplare il 12.5.2012 a Rovagnate.

\section{Lachnaia (Lachnaia) italica (Weise, 1882)}

Corotipo: S-Europeo (SEU).

Presenza in Italia: tutte le regioni.

Piante ospiti: Rosaceae (Rubus, Rosa), Fagaceae (Quercus).Occasionalmente dannoso alle colture di susine (Prunus salicinus) e ai giovani impianti forestali di querce (Regalin et al., 2006).
Osservazioni: la specie è stata reperita in 3 stazioni di campionamento, in un totale di 12 esemplari (Rovagnate, Squallera, Vertaggera).

Clytra (Clytra) laeviuscula Ratzeburg, 1837

Corotipo: Centroasiatico-Europeo (CAE).

Presenza in Italia: tutte le regioni, ad eccezione della Sardegna dove è sostituita da C. espanoli.

Piante ospiti: essenze arboree dei generi Salicaceae (Salix), Rosaceae (Crataegus, Prunus), Oleaceae (Fraxinus), Corylaceae (Corylus) e su Fabaceae (Dorycnium pentaphyllum*).

Osservazioni: la specie è stata reperita in 2 stazioni di campionamento, in un totale di 4 esemplari (Mondonico, Rovagnate).

Smaragdina affinis affinis (Illiger, 1794)

Corotipo: Europeo (EUR).

Presenza in Italia: Penisola.

Piante ospiti: Corylaceae (Corylus avellana), Fagaceae (Quercus), Rosaceae (Crataegus, Prunus spinosa*), Salicaceae (Salix, Populus), Ulmaceae (Ulmus), Vitaceae (Vitis vinifera*).

Osservazioni: la specie è stata reperita in 4 stazioni di campionamento, in un totale di 16 esemplari (Mondonico, Perego, Rovagnate, Squallera).

Smaragdina aurita aurita (Linné, 1767)

Corotipo: Sibirico-Europeo (SIE).

Presenza in Italia: Penisola.

Piante ospiti: Corylaceae (Corylus avellana*), Rosaceae (Crataegus, Prunus), Betulaceae (Alnus glutinosa, Betula), Fagaceae (Quercus).

Osservazioni: la specie è stata reperita in 2 stazioni di campionamento, in un totale di 2 esemplari (Perego, Rovagnate).

Smaragdina flavicollis (Charpentier, 1825)

Corotipo: Europeo (EUR).

Presenza in Italia: regioni settentrionali.

Piante ospiti: Betulaceae (Alnus glutinosa*), Corylaceae (Corylus).

Osservazioni: la specie è stata reperita in 3 stazioni di campionamento, in un totale di 3 esemplari (Rovagnate, Squallera, Valle S. Croce).

Smaragdina salicina (Scopoli, 1763)

Corotipo: Europeo (EUR).

Presenza in Italia: Penisola. Presenza da confermare in Sicilia.

Piante ospiti: Polygonaceae (Rumex), Rosaceae (Crataegus), Salicaceae (Salix), Corylaceae (Corylus).

Osservazioni: la specie è stata reperita in 4 stazioni di campionamento, in un totale di 18 esemplari (Perego, Rovagnate, Squallera, Vertaggera).

Coptocephala unifasciata unifasciata (Scopoli, 1763)

Corotipo: Centroasiatico-Europeo (CAE).

Presenza in Italia: Penisola e Sicilia.

Piante ospiti: Apiaceae (Echinophora, Pastinaca, 
Daucus, Ferulago, Peucedanum). Segnalata anche su Fabaceae (Sarothamnus).

Osservazioni: specie rinvenuta in un totale di 13 esemplari sui prati magri di Perego e Rovagnate.

Pachybrachis (Pachybrachis) hieroglyphicus (Laicharting, 1781)

Corotipo: Sibirico-Europeo (SIE).

Presenza in Italia: Penisola, Sicilia.

Piante ospiti: Salicaceae (Salix, Populus), Betulaceae (Betula).

Osservazioni: sul territorio del Parco la specie è stata raccolta solo su Salix in luoghi umidi. Reperita in 3 stazioni di campionamento per un totale di 19 esemplari.

Pachybrachis (Pachybrachis) tesselatus tesselatus (Olivier, 1791)

Corotipo: S-Europeo (SEU).

Presenza in Italia: Penisola, Sicilia.

Piante ospiti: Fagaceae (Quercus), Salicaceae (Salix, Populus), Corylaceae (Corylus), Ericaceae (Erica).

Osservazioni: la specie è stata raccolta il 18.6.2013 su Quercus pubescens nei prati magri di Rovagnate in un totale di 2 esemplari.

Cryptocephalus (Burlinius) fulvus fulvus Goeze, 1777

Corotipo: W-Paleartico (WPA).

Presenza in Italia: tutte le regioni.

Piante ospiti: Lamiaceae, Asteraceae, Salicaceae, Fabaceae, Apiaceae, Guttiferae, Geraniaceae, Ericaceae, Rubiaceae. Specie polifaga, più frequente in genere sul tappeto erboso.

Osservazioni: la specie è stata reperita in 3 stazioni di campionamento, in un totale di 3 esemplari (Perego, Squallera, Vertaggera).

Cryptocephalus (Burlinius) labiatus (Linné, 1761)

Corotipo: Sibirico-Europeo (SIE).

Presenza in Italia: Penisola e Sicilia.

Piante ospiti: Salicaceae (Salix, Populus nigra*, Populus alba*), Corylus spp., Fagaceae (Quercus pubescens*), Betulaceae (Betula pendula*, Alnus incana), Myricaceae (Myrica gale), Ericaceae (Vaccinium myrtillus*), Rosaceae (Fragaria). Specie polifaga, legata prevalentemente allo strato arboreo-arbustivo.

Osservazioni: sul territorio la specie è stata raccolta solo a Rovagnate, in un totale di 2 esemplari: 12.5.2012 e 18.VI.13.

Cryptocephalus (Burlinius) pygmaeus vittula Suffrian, 1848

Corotipo: S-Europeo (SEU).

Presenza in Italia: Penisola e Sicilia.

Piante ospiti: Lamiaceae (Origanum, Thymus, Satureja, Mentha).

Osservazioni: la specie è stata reperita in 2 stazioni di campionamento, in un totale di 3 esemplari (Landriano, Squallera).

Cryptocephalus (Burlinius) strigosus Germar, 1824

Corotipo: S-Europeo (SEU).
Presenza in Italia: regioni peninsulari, fino alla Campania.

Piante ospiti: Lamiaceae (Thymus), Betulaceae (Alnus).

Osservazioni: la specie è stata reperita in 3 stazioni di campionamento, in un totale di 12 esemplari (Perego, Rovagnate, Vertaggera).

Cryptocephalus (Cryptocephalus) bipunctatus (Linné, 1758)

Corotipo: Sibirico-Europeo (SIE).

Presenza in Italia: Penisola.

Piante ospiti: Salicaceae, Betulaceae, Corylaceae, Fagaceae, Rosaceae, Fabaceae.

Osservazioni: specie reperita ai margini del bosco termofilo nelle stazioni di Perego e Rovagnate in un totale di 9 esemplari.

Cryptocephalus (Cryptocephalus) flavipes Fabricius, 1781

Corotipo: Sibirico-Europeo (SIE).

Presenza in Italia: Penisola.

Piante ospiti: Salicaceae (Salix, Populus), Betulaceae (Betula pendula*, Alnus), Corylaceae (Corylus avellana*), Fagaceae (Quercus), Cistaceae, Rosaceae (Crataegus).

Osservazioni: specie reperita in numerosi esemplari solo sui prati aridi di Perego e Rovagnate in un totale di 57 esemplari.

Cryptocephalus (Cryptocephalus) janthinus Germar, 1824

Corotipo: Sibirico-Europeo (SIE).

Presenza in Italia: tutte le regioni, da confermare per la Sardegna.

Piante ospiti: Poaceae (Phragmites communis), Lytraceae (Lythrum salicaria*), Betulaceae (Betula), Salicaceae, Primulaceae (Lysimachia vulgaris*).

Osservazioni: specie rinvenuta in un'unica stazione, Mondonico, in un totale di 3 esemplari su Lythrum salicaria.

Cryptocephalus (Cryptocephalus) moraei (Linné, 1758)

Corotipo: Sibirico-Europeo (SIE).

Presenza in Italia: tutte le regioni.

Piante ospiti: Guttiferae (Hypericum).

Osservazioni: la specie è stata reperita in 3 stazioni di campionamento, in un totale di 8 esemplari (Mondonico, Perego, Valle S. Croce).

Cryptocephalus (Cryptocephalus) parvulus O.F. Müller, 1776

Corotipo: Sibirico-Europeo (SIE).

Presenza in Italia: Penisola e Sardegna.

Piante ospiti: Betulaceae (Betula pendula*), Salicaceae (Salix caprea*), Fagaceae (Quercus robur*), Corylaceae, Rosaceae (Crataegus laevigata*).

Osservazioni: specie rinvenuta solo a Mondonico in un totale di 3 esemplari.

Cryptocephalus (Cryptocephalus) transiens Franz, 1949 Corotipo: Centroeuropeo (CEU). 
Presenza in Italia: regioni settentrionali.

Piante ospiti: Asteraceae, Guttiferae, Ranunculaceae, Rutaceae, Fabaceae.

Osservazioni: nel Parco del Curone la specie è più frequente su Lotus corniculatus. Reperita in 8 stazioni di campionamento in un totale di 33 esemplari (Calendone, Landriano, Mondonico, Perego, Rovagnate, Squallera, Valle S. Croce, Vertaggera).

Hispa atra Linné, 1767

Corotipo: W-Paleartico (WPA).

Presenza in Italia: tutte le regioni.

Piante ospiti: Poaceae.

Osservazioni: la specie è stata reperita in 2 stazioni di campionamento, in un totale di 5 esemplari (Landriano, Squallera).

Hypocassida subferruginea (Schrank, 1776)

Corotipo: Paleartico (PAL).

Presenza in Italia: tutte le regioni.

Piante ospiti: Convolvulaceae (Convolvulus, Calystegia), Chenopodiaceae (Beta).

Osservazioni: la specie è stata reperita in 3 stazioni di campionamento, in un totale di 6 esemplari (Cà del Soldato, Mondonico, Vertaggera).

Cassida prasina Illiger, 1798

Corotipo: Paleartico (PAL).

Presenza in Italia: tutte le regioni eccetto la Sardegna.

Piante ospiti: Asteraceae (Achillea millefolium*, Matricaria, Artemisia, Anthemis).

Osservazioni: specie reperita in un unico esemplare il 13.5.2013 sui prati magri di Perego.

Cassida vibex Linné, 1767

Corotipo: Sibirico-Europeo (SIE).

Presenza in Italia: regioni settentrionali e centrali fino all'Umbria, Basilicata e Calabria (Sassi \& Borowiec, 2006).

Piante ospiti: Asteraceae (Cirsium palustre, C. arven$s^{*}$, Tanacetum vulgare*, Carduus, Centaurea jacea*, Arctium, Tanacetum, Achillea), Chenopodiaceae (Beta vulgaris*).

Osservazioni: la specie è stata reperita in 2 stazioni di campionamento, in un totale di 2 esemplari (Cà del Soldato, Squallera).

Cassida margaritacea Schaller, 1783

Corotipo: Sibirico-Europeo (SIE).

Presenza in Italia: Penisola e Sicilia.

Piante ospiti: Caryophyllaceae (Silene vulgaris*, S. dioica*, Saponaria officinalis*, Spergula arvensis*, Petrorhagia prolifera*), Lamiaceae (Thymus serpyllum), Asteraceae (Centaurea, Anaphalis margaritacea, Helichrysum arenarium).

Osservazioni: specie reperita in 2 esemplari il 23.8.2013 sui prati magri di Vertaggera.

Cassida viridis Linné, 1758

Corotipo: Asiatico-Europeo (ASE).

Presenza in Italia: tutte le regioni.
Piante ospiti: Lamiaceae (Stachys recta*, Mentha aquatica*, Galeopsis tetrahit*, Lycopus europaeus*, Salvia, Melissa, Cirsium).

Osservazioni: specie reperita il 18.6.2013 in un unico esemplare nella stazione di Perego, lungo il sentiero.

\section{DISCUSSIONE}

\section{Considerazioni ecologiche}

Per cercare di definire le relazioni esistenti tra le specie di Crisomelidi raccolte e la vegetazione del Parco, le piante ospiti/nutrici (limitandosi a quelle presenti nell'area di studio) sono state inquadrate (Tab. 3) in quattro categorie fitosociologiche (Fogato \& Leonardi, 1980): vegetazione xerofila (Festuco-Brometea), vegetazione mesofila (Querco-Fagetea), vegetazione igrofila (Molinia-Juncetea) o elofitica (Fragmitetea), vegetazione di influenza antropica (ruderale, prati da sfalcio, prati calpestati, colture).

Tab. 3 - Lista delle specie di Crisomelidi censiti e loro piante ospiti raggruppate in quattro categorie fitosociologiche. Legenda: Xero) vegetazione xerofila (classe FestucoBrometea), Igr) vegetazione igrofila (classe Molinia-Juncetea) o elofitica (classe Fragmitetea), Mes) vegetazione mesofila (classe Querco-Fagetea), Antr) vegetazione di influenza antropica.

\begin{tabular}{|l|c|c|c|c|}
\hline Specie & Xero & Igr & Mes & Antr \\
\hline Crioceris duodecimpunctata & $\mathrm{X}$ & & & \\
\hline Lema cyanella & $\mathrm{X}$ & & & \\
\hline Oulema duftschmidi & $\mathrm{X}$ & $\mathrm{X}$ & & $\mathrm{X}$ \\
\hline Oulema mauroi & $\mathrm{X}$ & & & \\
\hline Timarcha nicaeensis & $\mathrm{X}$ & & & $\mathrm{X}$ \\
\hline Chrysolina rossia & $\mathrm{X}$ & & & $\mathrm{X}$ \\
\hline Chrysolina fastuosa & & & $\mathrm{X}$ & \\
\hline Chrysolina herbacea & $\mathrm{X}$ & & & \\
\hline Chrysolina grossa & $\mathrm{X}$ & & & \\
\hline Chrysolina americana & $\mathrm{X}$ & & & \\
\hline Chrysolina haemoptera & $\mathrm{X}$ & & & $\mathrm{X}$ \\
\hline Chrysolina marginata & $\mathrm{X}$ & & & \\
\hline Chrysolina geminata & $\mathrm{X}$ & $\mathrm{X}$ & & \\
\hline Leptinotarsa decemlineata & & & & $\mathrm{X}$ \\
\hline Chrysomela populi & & & & $\mathrm{X}$ \\
\hline Chrysomela vigintipunctata & & $\mathrm{X}$ & & \\
\hline Linaeidea aenea & & & $\mathrm{X}$ & \\
\hline Galerucella lineola & & $\mathrm{X}$ & $\mathrm{X}$ & $\mathrm{X}$ \\
\hline Galerucella pusilla & $\mathrm{X}$ & $\mathrm{X}$ & & \\
\hline Galeruca pomonae & $\mathrm{X}$ & $\mathrm{X}$ & & $\mathrm{X}$ \\
\hline Galeruca tanaceti & $\mathrm{X}$ & $\mathrm{X}$ & & $\mathrm{X}$ \\
\hline Ophraella communa & $\mathrm{X}$ & & & $\mathrm{X}$ \\
\hline Diabrotica virgifera & & & & $\mathrm{X}$ \\
\hline
\end{tabular}




\begin{tabular}{|c|c|c|c|c|}
\hline Specie & Xero & Igr & Mes & Antr \\
\hline Calomicrus circumfusus & $\mathrm{X}$ & & & \\
\hline Luperus flavipes & $\mathrm{X}$ & & $\mathrm{X}$ & \\
\hline Luperus leonardii & & & $X$ & \\
\hline Luperus longicornis & $\mathrm{X}$ & $\mathrm{X}$ & & $\mathrm{X}$ \\
\hline Phyllotreta atra & $\mathrm{X}$ & & & \\
\hline Phyllotreta ochripes & & & & $\mathrm{X}$ \\
\hline Phyllotreta punctulata & $\mathrm{X}$ & & & \\
\hline Phyllotreta vittula & $X$ & $\mathrm{X}$ & & $\mathrm{X}$ \\
\hline Aphthona herbigrada & $\mathrm{X}$ & & & \\
\hline Aphthona lutescens & & $\mathrm{X}$ & & \\
\hline Aphthona ovata & $\mathrm{X}$ & & & \\
\hline Aphthona sicelidis & $X$ & & & \\
\hline Aphthona venustula & & $\mathrm{X}$ & & \\
\hline Longitarsus apicalis & & $X$ & & \\
\hline Longitarsus codinai & $\mathrm{X}$ & & & \\
\hline Longitarsus helvolus & $X$ & & & \\
\hline Longitarsus lewisii & $\mathrm{X}$ & & & $\mathrm{X}$ \\
\hline Longitarsus luridus & $\mathrm{X}$ & $\mathrm{X}$ & & $\mathrm{X}$ \\
\hline Longitarsus melanocephalus & $X$ & $X$ & & $X$ \\
\hline Longitarsus obliteratus & $\mathrm{X}$ & & & \\
\hline Longitarsus pellucidus & $X$ & & & $\mathrm{X}$ \\
\hline Longitarsus pratensis & $\mathrm{X}$ & $\mathrm{X}$ & & $\mathrm{X}$ \\
\hline Longitarsus salviae & $\mathrm{X}$ & & & \\
\hline Longitarsus succineus & $X$ & $\mathrm{X}$ & & $\mathrm{X}$ \\
\hline Altica brevicollis & & & $\mathrm{X}$ & \\
\hline Altica oleracea & $X$ & $\mathrm{X}$ & $\mathrm{X}$ & $\mathrm{X}$ \\
\hline Lythraria salicariae & & $\mathrm{X}$ & & \\
\hline Crepidodera aurata & & $\mathrm{X}$ & & \\
\hline Crepidodera fulvicornis & & $\mathrm{X}$ & & \\
\hline Podagrica malvae & $X$ & $\mathrm{X}$ & & \\
\hline Chaetocnema concinna & $\mathrm{X}$ & $\mathrm{X}$ & & $\mathrm{X}$ \\
\hline Chaetocnema conducta & $X$ & $\mathrm{X}$ & & $\mathrm{X}$ \\
\hline Chaetocnema hortensis & $\mathrm{X}$ & $\mathrm{X}$ & & $\mathrm{X}$ \\
\hline Chaetocnema picipes & $X$ & & & $\mathrm{X}$ \\
\hline Chaetocnema tibialis & $\mathrm{X}$ & $\mathrm{X}$ & & \\
\hline Sphaeroderma rubidum & $\mathrm{X}$ & $\mathrm{X}$ & & $\mathrm{X}$ \\
\hline Dibolia femoralis & $X$ & & & \\
\hline Dibolia rugulosa & $X$ & & & \\
\hline Psylliodes cuprea & $X$ & & & \\
\hline Psylliodes gibbosus & $\mathrm{X}$ & $\mathrm{X}$ & & \\
\hline Psylliodes napi & $\mathrm{X}$ & $\mathrm{X}$ & & $\mathrm{X}$ \\
\hline Labidostomis humeralis & $X$ & & & \\
\hline Labidostomis longimana & & $\mathrm{X}$ & & \\
\hline Labidostomis lucida & $X$ & & & \\
\hline Lachnaia italica & $X$ & & & \\
\hline Clytra laeviuscula & $\mathrm{X}$ & $\mathrm{X}$ & $\mathrm{X}$ & $\mathrm{X}$ \\
\hline Smaragdina affinis & $\mathrm{X}$ & $\mathrm{X}$ & $X$ & \\
\hline
\end{tabular}

\begin{tabular}{|l|c|c|c|c|}
\hline Specie & Xero & Igr & Mes & Antr \\
\hline Smaragdina aurita & $\mathrm{X}$ & & & \\
\hline Smaragdina flavicollis & $\mathrm{X}$ & $\mathrm{X}$ & & \\
\hline Smaragdina salicina & $\mathrm{X}$ & & & \\
\hline Coptocephala unifasciata & $\mathrm{X}$ & & & \\
\hline Pachybrachis hieroglyphicus & & $\mathrm{X}$ & & \\
\hline Pachybrachis tesselatus & $\mathrm{X}$ & & & \\
\hline Cryptocephalus fulvus & $\mathrm{X}$ & & & \\
\hline Cryptocephalus labiatus & $\mathrm{X}$ & & $\mathrm{X}$ & \\
\hline Cryptocephalus pygmaeus & & $\mathrm{X}$ & & $\mathrm{X}$ \\
\hline Cryptocephalus strigosus & $\mathrm{X}$ & & & \\
\hline Cryptocephalus bipunctatus & $\mathrm{X}$ & & $\mathrm{X}$ & \\
\hline Cryptocephalus flavipes & $\mathrm{X}$ & & $\mathrm{X}$ & \\
\hline Cryptocephalus janthinus & & $\mathrm{X}$ & & \\
\hline Cryptocephalus moraei & $\mathrm{X}$ & $\mathrm{X}$ & & $\mathrm{X}$ \\
\hline Cryptocephalus parvulus & & $\mathrm{X}$ & $\mathrm{X}$ & \\
\hline Cryptocephalus transiens & $\mathrm{X}$ & $\mathrm{X}$ & & $\mathrm{X}$ \\
\hline Hispa atra & & & & $\mathrm{X}$ \\
\hline Hypocassida subferruginea & $\mathrm{X}$ & $\mathrm{X}$ & & $\mathrm{X}$ \\
\hline Cassida prasina & $\mathrm{X}$ & & & \\
\hline Cassida vibex & $\mathrm{X}$ & $\mathrm{X}$ & & \\
\hline Cassida margaritacea & $\mathrm{X}$ & & & \\
\hline Cassida viridis & $\mathrm{X}$ & & & \\
\hline
\end{tabular}

\section{Fenologia}

Come risulta dalla Fig. 10, il numero di individui catturato in marzo e durante i mesi autunnali (ottobre e novembre) è basso anche se mai nullo. Da aprile si assiste ad un incremento delle catture che si accentua nei mesi estivi. A settembre si assiste ad un repentino calo. Con l'avanzare della stagione fredda, si ha un nuovo decremento fino ai già ricordati minimi autunnali.

Il numero di specie segue un andamento molto simile a quello degli individui, se si eccettua un netto incremento da aprile a maggio. Inoltre il numero di specie raggiunge i livelli massimi in giugno, per poi diminuire in luglio e agosto. Il numero di specie raccolte è decisamente basso

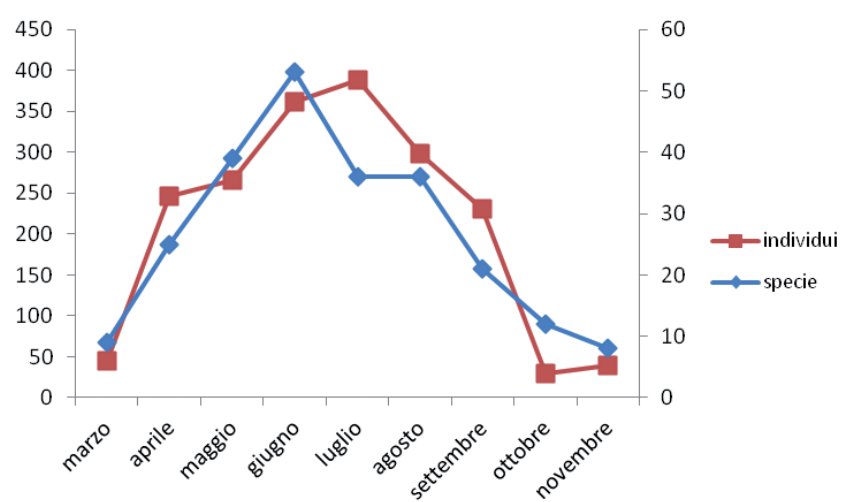

Fig. 10 - Fenologia complessiva delle specie e degli individui catturati durante i campionamenti per tutto il territorio del Parco. 
nei mesi autunnali. Questo potrebbe essere dovuto anche al fatto che le uscite in ottobre e novembre sono state limitate solo ad alcune stazioni.

In generale non si registra un notevole calo, durante la stagione estiva, né delle specie né degli individui.

Nella Tab. 4 viene riportata infine la ripartizione mensile del numero di esemplari per ciascuna specie presente nel Parco. La tabella rappresenta un semplice quadro orientativo dell'abbondanza delle specie nelle raccolte condotte nei diversi periodi dell'anno. Per ogni specie sono indicate le stazioni di presenza (st), il numero degli esemplari raccolti per ogni stazione nei diversi periodi dell'anno e il numero complessivo degli esemplari.

Tab. 4 - Indicazione del numero di esemplari catturati per mese per ciascuna specie.

Legenda: A) prima quindicina del mese. $\mathrm{B}=$ seconda quindicina del mese.

st $=$ stazione di presenza. Per $=$ Perego; Rov $=$ Rovagnate $;$ Mon $=$ Mondonico $;$ Cal $=$ Calendone;

Cas $=$ Cà del Soldato; Ver $=$ Vertaggera; Squ $=$ Squallera; Vsc $=$ Valle S. Croce; Lan $=$ Landriano.

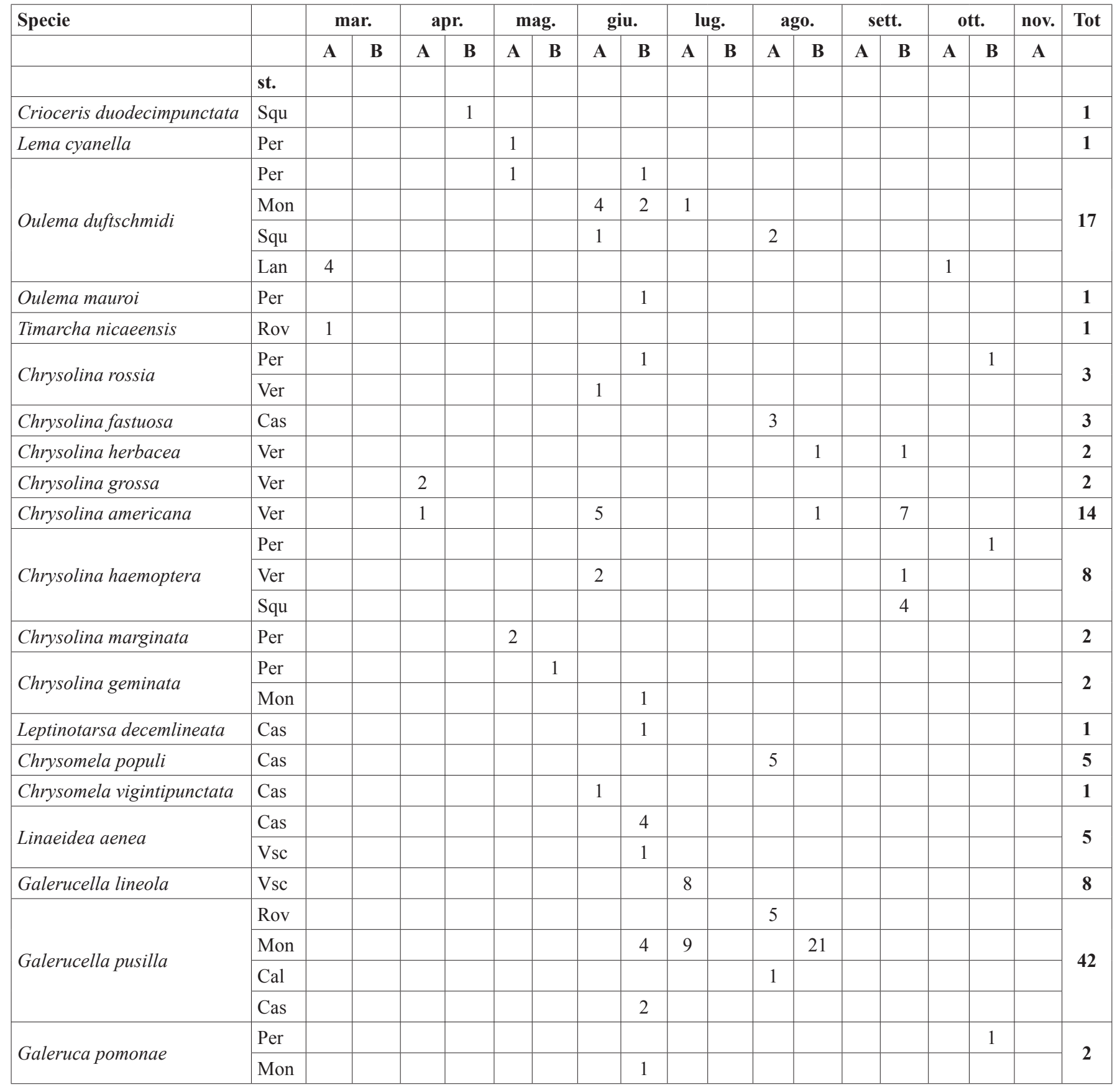




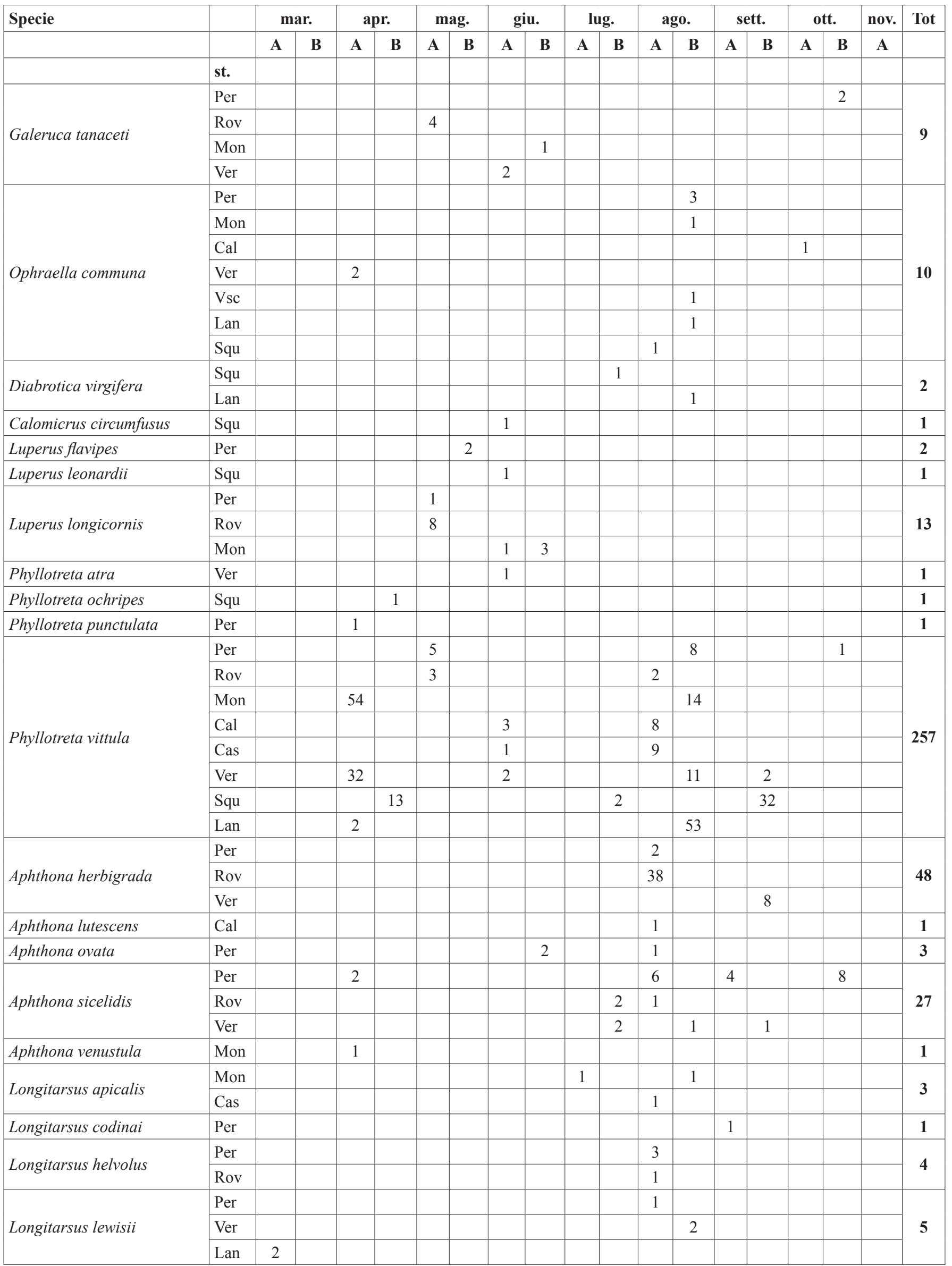




\begin{tabular}{|c|c|c|c|c|c|c|c|c|c|c|c|c|c|c|c|c|c|c|c|}
\hline \multirow[t]{2}{*}{ Specie } & & \multicolumn{2}{|c|}{ mar. } & \multicolumn{2}{|c|}{ apr. } & \multicolumn{2}{|c|}{ mag. } & \multicolumn{2}{|c|}{ giu. } & \multicolumn{2}{|c|}{ lug. } & \multicolumn{2}{|c|}{ ago. } & \multicolumn{2}{|c|}{ sett. } & \multicolumn{2}{|c|}{ ott. } & \multirow{2}{*}{\begin{tabular}{|c|} 
nov. \\
A \\
\end{tabular}} & \multirow[t]{2}{*}{ Tot } \\
\hline & & $\mathbf{A}$ & B & $\mathbf{A}$ & B & A & B & $\mathbf{A}$ & B & A & B & $\mathbf{A}$ & B & $\mathbf{A}$ & B & $\mathbf{A}$ & B & & \\
\hline & st. & & & & & & & & & & & & & & & & & & \\
\hline \multirow{5}{*}{ Longitarsus luridus } & Rov & & & 1 & & 6 & & & & & 6 & & & & & & & & \multirow{5}{*}{100} \\
\hline & Mon & & & 4 & & & & & & 2 & & & 2 & & & & & 10 & \\
\hline & Ver & 3 & & 2 & & & & & & & 30 & & 3 & 1 & & & & & \\
\hline & Vsc & & & & & & & & & 2 & & & & & & & & & \\
\hline & Lan & & & & & & 1 & & & & & & & & & & & & \\
\hline \multirow{3}{*}{ Longitarsus melanocephalus } & Per & & & 4 & & & & & & & & & & & & & & & \multirow{3}{*}{27} \\
\hline & Squ & & 4 & & & & & & & & & & & & & & & & \\
\hline & Vsc & & & & & & & & & 5 & & & 1 & & & & & & \\
\hline Lonoitarsus ohliteratus & Per & & & & & & & & & & & 8 & & 2 & & & & & 20 \\
\hline Longilarsus oomeratus & Rov & & & & & 1 & & & & & 7 & 2 & & & & & & & 20 \\
\hline & Per & & & 1 & & 4 & & & & & & 1 & 5 & 2 & & & 2 & & \\
\hline Lonoitarsus nellucidus & Rov & & & & & & & & 1 & & 3 & 3 & & & & & & & 32 \\
\hline Longuarsus pelluctaus & Ver & & & 1 & & & & & & & & & 5 & & & & & & 32 \\
\hline & Vsc & & & & & & & & & 4 & & & & & & & & & \\
\hline & Per & & & 10 & & & & & & & & 44 & 5 & 6 & & & 2 & & \\
\hline & Lan & 4 & & & & & 1 & & & & & & 1 & & & & & & \\
\hline Lonoitarcus salvige & Per & & & 2 & & & & & & & & 10 & 2 & & & & & & 42 \\
\hline Longlarsus satviae & Rov & & & & & & & & 4 & & 22 & 2 & & & & & & & 42 \\
\hline & Per & & & 1 & & & & & 2 & & & 1 & & 6 & & & 9 & & \\
\hline & Rov & & & & & & & & 1 & & 1 & 9 & & & & & & 1 & \\
\hline & Mon & & & & & & & 1 & 1 & & & & & & & & & 2 & \\
\hline Longitarsus succineus & Cas & & & & & & & & & & & 2 & & & & & & & 63 \\
\hline Longliarsus succtneus & Ver & 1 & & & & & & & & & & & & & 11 & & & & 03 \\
\hline & Vsc & & & & & & & & & & & & 1 & & & & & & \\
\hline & $\mathrm{Squ}$ & & & & & & & & & & & & & & 3 & & & & \\
\hline & Lan & & & & & & 1 & & & & & & & & & & & & \\
\hline Altica brevicollis & Cas & & & & & & & 2 & 1 & & & & & & & & & & 3 \\
\hline & Per & & & & & & & & & & & 1 & 1 & & & & & & \\
\hline & Rov & & & & & 1 & & & & & 4 & & & & & & & & \\
\hline & Mon & & & 2 & & & & 1 & 6 & 2 & & & 11 & & & & & & \\
\hline Altica oleracea & Cal & & & & & & & & 3 & & & & & & & & & & 44 \\
\hline & Ver & & & 1 & & & & & & & & & 2 & & & & & & \\
\hline
\end{tabular}




\begin{tabular}{|c|c|c|c|c|c|c|c|c|c|c|c|c|c|c|c|c|c|c|c|}
\hline \multirow[t]{2}{*}{ Specie } & & \multicolumn{2}{|c|}{ mar. } & \multicolumn{2}{|c|}{ apr. } & \multicolumn{2}{|c|}{ mag. } & \multicolumn{2}{|c|}{ giu. } & \multicolumn{2}{|c|}{ lug. } & \multicolumn{2}{|c|}{ ago. } & \multicolumn{2}{|c|}{ sett. } & \multicolumn{2}{|c|}{ ott. } & \multirow{2}{*}{$\begin{array}{c}\text { nov. } \\
\text { A }\end{array}$} & \multirow[t]{2}{*}{ Tot } \\
\hline & & A & B & A & B & $\mathbf{A}$ & B & A & B & A & B & A & B & A & B & A & B & & \\
\hline & st. & & & & & & & & & & & & & & & & & & \\
\hline \multirow{2}{*}{ Lythraria salicariae } & Mon & & & & & & & & 1 & & & & & & & & & & \multirow{2}{*}{15} \\
\hline & $\mathrm{CaS}$ & & & & & & & 1 & 13 & & & & & & & & & & \\
\hline \multirow{7}{*}{ Crepidodera aurata } & Per & & & & & & & & & 2 & & & & 1 & & & & & \\
\hline & Rov & & & & & 1 & & & & & & & & & & & & & \\
\hline & Mon & & & & & & & & 9 & 2 & & 6 & & & & & & 2 & \\
\hline & $\mathrm{Cal}$ & & & & & & & & 8 & & & 2 & & & & & & & 171 \\
\hline & $\mathrm{CaS}$ & & & & & & & 4 & 27 & & & & & & & & & & \\
\hline & Squ & & & & 5 & & & 35 & & & 7 & 2 & & & 13 & & & & \\
\hline & Vsc & & & & & & & & 41 & 4 & & & & & & & & & \\
\hline Crepidodera fulvicornis & $\mathrm{Cal}$ & & & & & & & & 8 & & & 1 & & & & & & & 9 \\
\hline 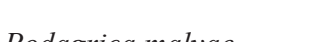 & Per & & & 1 & & & & & & & & & & & & & & & 2 \\
\hline 1 tangrac a matrat & Mon & & & 1 & & & & & & & & & & & & & & & 2 \\
\hline & Mon & & & 7 & & & & & 1 & 2 & & & & & & & & & \\
\hline Chaetocnema concinna & Squ & & & & 2 & & & & & & & & & & & & & & 12 \\
\hline & Cal & & & & & & & 1 & & & & & & & & & & & \\
\hline & Per & & & & & & & & & & & & & 2 & & & & & \\
\hline & Rov & & & & & 4 & & & & & & 1 & & & & & & & \\
\hline & Mon & & & 3 & & & & & 4 & 1 & & 4 & & & & & & & \\
\hline & $\mathrm{Cal}$ & & & & & & & 7 & & & & 1 & & & & & & & \\
\hline Chaetocnema conducta & $\mathrm{CaS}$ & & & & & & & & & & & 2 & & & & & & & 88 \\
\hline & Ver & & & 3 & & & & 2 & & & & & 1 & & 5 & & & & \\
\hline & Squ & & & & & & & 2 & & & 1 & 2 & & & 33 & & & & \\
\hline & Vsc & & & & & & & & & & & & 3 & 1 & & & & & \\
\hline & Lan & & & & & & 4 & & & & & & 1 & & & 1 & & & \\
\hline & Per & & & 1 & & & & & & & & & & 2 & & & & & \\
\hline & Rov & & & 1 & & 1 & & & & & & & & & & & & & \\
\hline & Mon & & & 54 & & & & 5 & & 3 & & & & & & & & & \\
\hline & $\mathrm{Cal}$ & & & & & & & 1 & & & & & & & & & & & \\
\hline Chaetocnema hortensis & $\mathrm{CaS}$ & & & & & & & & 1 & & & 2 & & & & & & & 119 \\
\hline & Ver & 1 & & 1 & & & & 1 & & & & & 1 & & 5 & & & & \\
\hline & Squ & & & & 1 & & & & & & & 2 & & & 31 & & & & \\
\hline & Vsc & & & & & & & & & 2 & & & 1 & & & & & & \\
\hline & Lan & 2 & & & & & & & & & & & & & & & & & \\
\hline & Per & & & & & 1 & & & & & & & & & & & & & \\
\hline Chaetocnema picipes & Rov & & & & & & & & & & & 1 & & & & & & & 7 \\
\hline chaelocnema pictpes & Ver & & & 2 & & & & & & & & & & & & & & & 1 \\
\hline & Cal & & & & & & & 1 & & & & 1 & & & & & & & \\
\hline & Mon & & & 1 & & & & 1 & & 2 & & 1 & & & & & & & \\
\hline Chaetocnema tibialis & Ver & & & 1 & & & & & & & & & & & & & & & 8 \\
\hline & Squ & & & & & & & & & & & 2 & & & & & & & \\
\hline & Per & & & & & 1 & & & 9 & & & & 5 & 19 & & & & & \\
\hline & Rov & & & & & 1 & & & 2 & & 1 & & & & & & & & \\
\hline & Mon & & & & & & & 4 & 9 & & & & 3 & & & & & 3 & \\
\hline Sphaeroderma rubidum & Cas & & & & & & & 1 & & & & & & & & & & & 96 \\
\hline & Ver & & & & & & & 7 & & & & & 3 & & 7 & & & & \\
\hline & Squ & & & & & & & 17 & & & & 2 & & & 1 & & & & \\
\hline & Vsc & & & & & & & & & & & & & 1 & & & & & \\
\hline
\end{tabular}




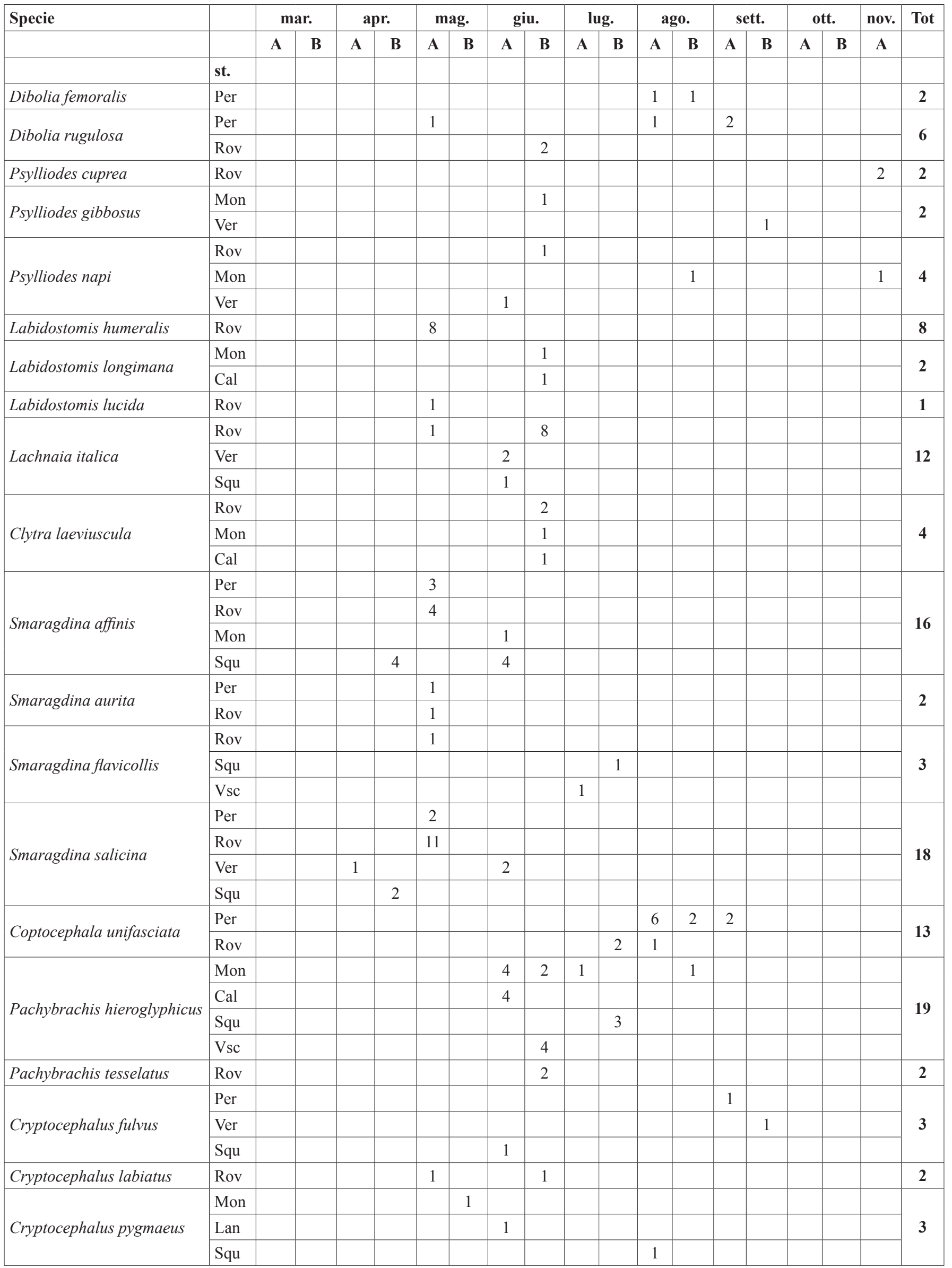




\begin{tabular}{|c|c|c|c|c|c|c|c|c|c|c|c|c|c|c|c|c|c|c|c|}
\hline \multirow[t]{2}{*}{ Specie } & & \multicolumn{2}{|c|}{ mar. } & \multicolumn{2}{|c|}{ apr. } & \multicolumn{2}{|c|}{ mag. } & \multicolumn{2}{|c|}{ giu. } & \multicolumn{2}{|c|}{ lug. } & \multicolumn{2}{|c|}{ ago. } & \multicolumn{2}{|c|}{ sett. } & \multicolumn{2}{|c|}{ ott. } & \multirow{2}{*}{$\begin{array}{c}\text { nov. } \\
\text { A }\end{array}$} & \multirow[t]{2}{*}{ Tot } \\
\hline & & $\mathbf{A}$ & B & A & B & A & B & $\mathbf{A}$ & B & $\mathbf{A}$ & B & $\mathbf{A}$ & B & $\mathbf{A}$ & B & $\mathbf{A}$ & B & & \\
\hline & st. & & & & & & & & & & & & & & & & & & \\
\hline \multirow{3}{*}{ Cryptocephalus strigosus } & Per & & & & & & & & & & & 2 & 1 & & & & & & \multirow{3}{*}{12} \\
\hline & Rov & & & & & & & & 3 & & 1 & & & & & & & & \\
\hline & Ver & & & & & & & & & & 4 & & 1 & & & & & & \\
\hline \multirow{2}{*}{ Cryptocephalus bipunctatus } & Per & & & & 1 & 2 & 1 & & & & & & & & & & & & \multirow{2}{*}{9} \\
\hline & Rov & & & & & 5 & & & & & & & & & & & & & \\
\hline \multirow{3}{*}{ Cryptocephalus flavipes } & Per & & & & 4 & 5 & 20 & & & & & & & & & & & & \multirow{3}{*}{57} \\
\hline & Rov & & & & & 21 & & & 6 & & & & & & & & & & \\
\hline & Ver & & & & & & & 1 & & & & & & & & & & & \\
\hline Cryptocephalus janthinus & Mon & & & & & & & & 3 & & & & & & & & & & 3 \\
\hline \multirow{4}{*}{ Cryptocephalus moraei } & Per & & & & & & & & & & & 1 & & & & & & & \multirow{4}{*}{8} \\
\hline & Mon & & & & & & & & 2 & & & & & & & & & 1 & \\
\hline & Cas & & & & & & & 1 & & & & & & & & & & & \\
\hline & Vsc & & & & & & & & & 3 & & & & & & & & & \\
\hline Cryptocephalus parvulus & Mon & & & & & & & & 1 & & & & 2 & & & & & & 3 \\
\hline \multirow{8}{*}{ Cryptocephalus transiens } & Per & & & & 1 & & & & & & & & & & & & & & \multirow{8}{*}{33} \\
\hline & Rov & & & & & 1 & & & 2 & & & 1 & & & & & & & \\
\hline & Mon & & & & & & & 1 & 3 & & & & & & & & & & \\
\hline & Cal & & & & & & & & 1 & & & & & & & & & & \\
\hline & Ver & & & & & & & 3 & & & 6 & & 1 & & & & & & \\
\hline & Squ & & & & & & & 2 & & & 2 & 4 & & & & & & & \\
\hline & Vsc & & & & & & & & & 4 & & & & & & & & & \\
\hline & Lan & & & & & & & & & & & & 1 & & & & & & \\
\hline \multirow{2}{*}{ Hispa atra } & Lan & 3 & & & & & & & & & & & & & & & & & 5 \\
\hline & Squ & & & & & & & & & & & 2 & & & & & & & 3 \\
\hline & Mon & & & & & & & & 1 & & & & & & & & & & \\
\hline Hypocassida subferruginea & Cas & & & & & & & & & & & 1 & & & & & & & 6 \\
\hline & Ver & & & & & & & 3 & & & & & 1 & & & & & & \\
\hline Cassida prasina & Per & & & & & 1 & & & & & & & & & & & & & 1 \\
\hline Cassida viher & Cas & & & & & & & & & & & 1 & & & & & & & 2 \\
\hline Cassida vibex & Squ & & & & 1 & & & & & & & & & & & & & & 2 \\
\hline Cassida margaritacea & Ver & & & & & & & & & & & & 2 & & & & & & 2 \\
\hline Cassida viridis & Per & & & & & & & & 1 & & & & & & & & & & 1 \\
\hline
\end{tabular}

\section{CONCLUSIONI}

Il totale delle specie registrate per il territorio in studio è di 92 unità. Questo contingente rappresenta il 15\% di tutte le specie accertate per il nord Italia e il 10\% dell'intera fauna crisomelidologica italiana (Biondi et al., 1994; Löbl \& Smetana, 2010).

Le specie numericamente predominanti nello strato erbaceo sono Phyllotreta vittula, Longitarsus luridus, Longitarsus pratensis e Chaetocnema hortensis, nello strato arboreo Crepidodera aurata e Cryptocephalus flavipes.

Le seguenti specie, tendenzialmente xerofile, sono caratteristiche dei prati magri del Parco: Chrysolina americana, Aphthona sicelidis, Longitarsus codinai, Longitarsus helvolus, Longitarsus obliteratus, Longitarsus salviae,
Dibolia femoralis, Dibolia rugulosa, Coptocephala unifasciata, Pachybrachis tesselatus, Cassida margaritacea.

Le seguenti specie, igrofile o igro-mesofile, sono state rinvenute in aree umide: Chrysomela vigintipunctata, Galerucella pusilla, Aphthona lutescens, Longitarsus apicalis, Crepidodera aurata, Crepidodera fulvicornis, Lythraria salicariae, Pachybrachis hieroglyphicus, Cryptocephalus janthinus, Cassida viridis.

Segnalo inoltre la presenza di Labidostomis lucida, specie tendenzialmente orofila, presente in Lombardia solo in ambito montano e alpino (Regalin, 1981). Potrebbe trattarsi di un elemento a carattere relittuale, legata ad aree aperte (prati) quando in epoca preistorica questa zona era ricoperta da foreste (Regalin in verbis). 
La ricchezza di specie è risultata più alta nelle stazioni di Perego (52\%) e Rovagnate (41\%), dove le raccolte sono state effettuate principalmente sulle praterie xerotermiche terrazzate. Le raccolte sui prati umidi di Mondonico hanno fruttato il $37 \%$ delle specie. Le stazioni di Vertaggera e Squallera, nelle quali le praterie xerotermiche sono maggiormente alterate hanno comunque fatto rilevare un'interessante percentuale di specie: $36 \%$ e $32 \%$ rispettivamente. La ricchezza di specie si presenta più bassa nelle stazioni di Cà del Soldato (23\%), Valle S. Croce (17\%), Calendone (16\%), Landriano (15\%), dove l'impatto antropico è maggiore e ha contribuito all'alterazione degli habitat naturali.

Alcuni taxa riportati nel lavoro risultano di notevole interesse faunistico. Infatti tre specie sono nuove per la Lombardia: Aphthona sicelidis, Crepidodera fulvicornis, Dibolia femoralis.

L'analisi zoogeografica (Figg. 11-12) ha evidenziato che il gruppo corologico maggiormente rappresentato è costituito dalle specie ad ampia distribuzione nella regione Olartica $(62 \%)$, in cui prevale la componente asiaticoeuropea $(21 \%)$ e sibirico-europea (15\%). Significativa si rivela anche la presenza degli elementi europei (28\%), dove a livello dei singoli corotipi fondamentali risultano prevalere quelli europei $(13 \%)$ e sud europei $(11 \%)$. Le specie mediterranee sono rappresentate dal $6 \%$ delle specie, dimostrando anche la presenza di elementi più spiccatamente termofili.

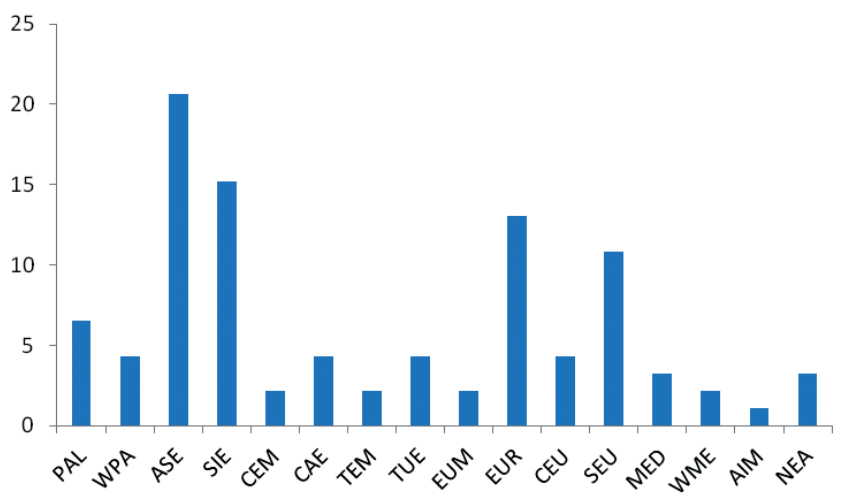

Fig. 11 - Percentuale di specie censite per ciascun corotipo fondamentale.

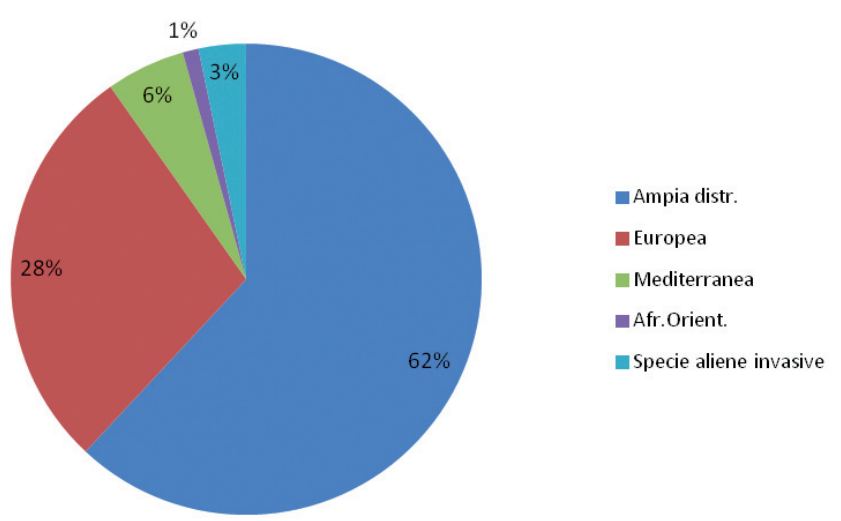

Fig. 12 - Corotipi raggruppati per categorie sintetiche.
La frequenza percentuale delle diverse sottofamiglie (Fig. 13) ricalca generalmente il pattern rilevato per le regioni settentrionali. Anche se le tecniche di raccolta utilizzate sono ritenute molto efficaci nel condurre campionamenti esaustivi di Coleotteri Crisomelidi (Sassi, 2007), è verosimile che lo spettro faunistico non sia stato completamente indagato. Ad esempio il numero di Chrysomelinae e di Cryptocephalini effettivamente presenti sul territorio potrebbe essere superiore a quello registrato, vista la percentuale di rinvenimenti piuttosto bassa rispetto al nord Italia (Löbl \& Smetana, 2010). Resta interessante notare come la percentuale di Clytrini e Galerucinae sia piuttosto alta rispetto a quella segnalata per il nord Italia (Löbl \& Smetana, 2010).

Il presente studio oltre a fornire l'elenco delle specie note per l'area in esame, vuole contribuire a porre l'attenzione anche sulla presenza, all'interno dell'area del Parco, di habitat non protetti che, vista la loro ricchezza di biodiversità, necessiterebbero di adeguate misure di tutela.

Sebbene la gestione a fini conservazionistici operata sul territorio del Parco garantisca un certo grado di tutela della fauna, sono tuttavia presenti alcuni fattori di rischio che minacciano lo stato di conservazione di molte specie di Insetti.

La cessazione dell'attività colturale su vaste superfici con substrato a scaglia rossa ha consentito negli scorsi decenni l'espandersi delle cenosi del Festuco-Brometalia. Tali cenosi sono peraltro coinvolte da processi evolutivi che conducono verso formazioni termofile a roverella e/o verso, o tramite, la fase successionale del Trifolio-Geranetea, e sono disturbate dalla ripresa delle attività agricole. Queste cenosi, presenti in particolare a Perego e a Rovagnate sono quelle in cui si è riscontrato il maggior numero di specie di Crisomelidi. Questi ambienti, ad alta diversità specifica, sono degni di essere tutelati e conservati. La Regione Lombardia, tramite il progetto Rete Natura 2000 - Bioitaly, ha posto l'attenzione sugli ambiti naturali, ma anche su quelli seminaturali e degradati, ma potenzialmente recuperabili (Cannone, 1996). Per la loro elevata ricchezza e complessità gli habitat dei prati magri del Parco sono stati proposti come Siti di Importanza Comunitaria. Si tratta di prati magri seminaturali

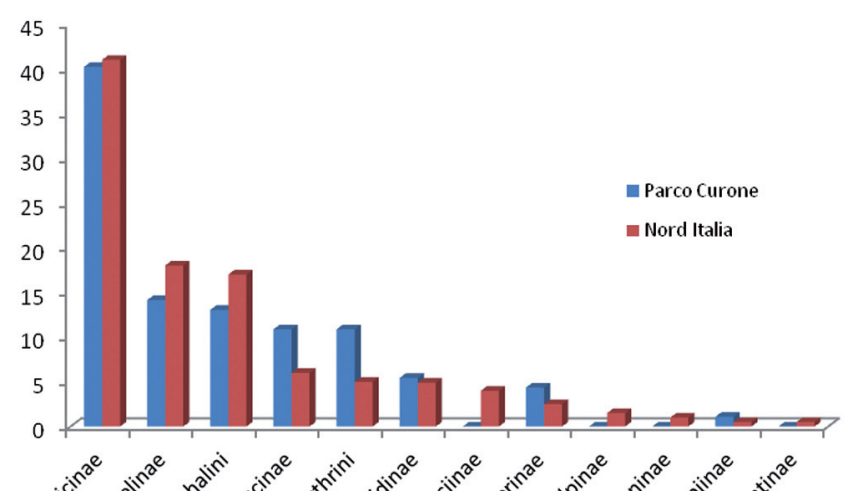

Fig. 13 - Distribuzione percentuale per sottofamiglie delle specie rilevate confrontata con i dati relativi al nord Italia (Löbl \& Smetana, 2010). 
particolarmente ricchi di specie, la cui presenza è legata ad un substrato di tipo calcareo e la cui conservazione è strettamente dipendente da una corretta gestione. I mancati interventi di sfalcio portano come conseguenza alla diminuzione del numero di specie a causa dei troppo elevati livelli di competizione, provocando la scomparsa delle specie di maggiore rilevanza naturalistica. A ciò segue il progressivo avanzamento del bosco.

Negli anni 2000-2003 è stato condotto un approccio gestionale ai prati magri del Parco nell'ambito di un progetto Life-Natura (Cereda \& Panseri, 2003). Alla realizzazione di interventi di conservazione attiva e recupero sono state associate azioni di indirizzo e complessivo governo dei cambiamenti nell'uso del suolo, per garantire il mantenimento e l'aumento dei valori ambientali e nel contempo la conduzione agricola in forme di elevata sostenibilità.

In questo senso il Parco ha inteso operare una serie di interventi atti ad assicurare il perpetrarsi di quelle pratiche agricole che permettono il mantenimento non solo di consorzi di vegetazione ad alta diversità specifica, ma anche delle stesse associazioni vegetali di riferimento, impedendo un ritorno massiccio del bosco. Gli interventi di manutenzione e recupero hanno avuto l'obbiettivo di conservare le condizioni ottimali che sussistono nelle superfici coltivate a prato prevenendone l'abbandono e negli incolti erbacei e recuperare superfici a densa copertura di rovi ed arbusti (Cereda, 2000). Gli interventi sono stati eseguiti attenendosi alle linee guida per la gestione dei prati magri, ma anche sperimentando l'efficacia di modalità diverse, con l'esecuzione di due tagli nell'anno ed il pascolo degli asini. Nel 2003 sono proseguiti gli interventi atti alla conservazione delle situazioni di maggiore interesse (Cereda \& Panseri, 2003). Sono state anche offerte indicazioni efficaci alle aziende agricole, in termini di alternative colturali, al fine di contribuire alla conservazione dei valori naturalistici ed ambientali. A tuttora la situazione conservativa si presenta abbastanza favorevole per quanto riguarda i prati magri di Perego. Non si può dire la stessa cosa per quelli di Rovagnate, dove l'abbandono delle pratiche di gestione ha portato negli ultimi anni a una veloce espansione di vegetazione forestale. Allo stesso tempo i prati magri di Squallera, abbandonati in parte, sono ora invasi da Agrimonia eupatoria, Rubus spp. e cespugli di Mentha spicata. I prati magri di Vertaggera, invece, sono tuttora sottosposti ad una certa pressione antropica dovuta alla presenza di coltivi, orti e frutteti.

Per quanto riguarda i prati umidi del Parco, nelle vicinanze degli stagni quali quello di Calendone, questi si presentano piuttosto degradati e in parte trasformati in colture o aree urbanizzate. Pur presentandosi in contesti più naturali, i prati di Mondonico, Cà del Soldato, Valle S. Croce e Landriano sono sottoposti ad una costante pressione antropica, dovuta alle coltivazioni e alla frequentazione turistica. Emerge l'importanza di recuperare delle aree umide per la conservazione di alcune specie igrofile.

In conclusione il Parco del Curone ospita una discreta ricchezza di fauna crisomelidologica, ma il numero di specie potrebbe essere più alto (per esempio, nonostante la presenza di abbondante fragmiteto lungo le sponde degli stagni, la sottofamiglia Donaciinae sembrerebbe non essere rappresentata da alcuna specie - Fig. 13). Il processo di erosione della biodiversità potrebbe continuare in futuro, se non saranno intraprese azioni volte alla conservazione e al ripristino di situazioni di abbandono gestionale. Per mantenere in buono stato di conservazione le popolazioni di Insetti fitofagi, tra cui i Coleotteri Crisomelidi, si ritiene quindi necessario realizzare ulteriori interventi finalizzati alla gestione e alla tutela degli habitat e alla pianificazione delle attività agricole. Sarebbe auspicabile la ripresa di un nuovo progetto di gestione che comprenda, oltre alla tutela dei prati magri, anche quella di altre aree prative del Parco.

\section{Ringraziamenti}

Sono molto grata all'amico e per me Maestro Carlo Leonardi che con la sua pazienza e cortesia mi ha seguito nella stesura di questo lavoro e mi ha aiutato nella realizzazione dei miei primi disegni entomologici. Ringrazio Carlo anche per i dati che mi ha fornito relativamente alle località di provenienza di esemplari di Aphthona sicelidis e Aphthona bonvouloiri del Museo Civico di Storia Naturale di Milano e del Naturhistorisches Museum di Basel.

Un sentito ringraziamento va a Valter Fogato per avermi gentilmente fornito le immagini degli edeagi e degli esemplari di Aphthona bonvouloiri e Aphthona sicelidis.

Sono grata agli amici Maurizio Biondi, Renato Regalin, Davide Sassi e Stefano Zoia per alcune determinazioni e per aver sempre risposto con sollecitudine alle mie domande.

\section{BIBLIOGRAFIA}

AA.VV., 2010 - Piano di gestione del SIC IT2030006 "Valle Santa Croce e Valle Curone". Parco Regionale di Montevecchia e della Valle del Curone, Montevecchia.

Balachowski A.S., 1963 - Entomologie appliquée à l'agriculture, Tome 1, vol. 2 (Phytophagoidea, suite et fin). Masson, Paris: 1-1391.

Berger W.H. \& Parker F.L., 1970 - Diversity of planktonic Foraminifera in deep sea sediments. Science 168, 1345-1347.

Bertolini S. de, 1886 - Catalogo sinonimico e topografico dei Coleotteri d'Italia, Firenze.

Bezděk J. \& Baselga, A., 2015 - Revision of western Palaearctic species of the Oulema melanopus group, with description of two new species from Europe (Coleoptera: Chrysomelidae: Criocerinae). Acta Entomologica, Musei Nationalis Pragae, 55 (1): 273-304.

Biondi M., 1990 - Elenco commentato dei Crisomelidi Alticini della fauna italiana (Coleoptera). Fragmenta Entomologica, Roma, 22 (1): 109-183.

Biondi M., Osella G.B., Zuppa A.M., 1992 - Studi zoologici sulla palude della Zittola (Abruzzo-Molise). III. Coleoptera Chrysomelidae. Rivista di Idrobiologia, 31: 51-93.

Biondi M., 1994 - Il popolamento a Coleoptera Chrysomelidae dell'Appennino umbro-marchigiano: considerazioni geografiche ed ecologiche. Biogeographia, 17 (1993): 321-365. 
Biondi M., Daccordi M., Regalin R., Zampetti M., 1994 - Coleoptera Polyphaga XV (Chrysomelidae, Bruchidae). In: Checklist delle specie della fauna italiana, 60. Minelli, A., Ruffo S. \& La Posta S. (eds.). Calderini, Bologna.

Biondi M., 2013 - Fauna Europaea: Chrysomelidae Alticinae. In: Fauna Europaea: Coleoptera, Beetles. Audisio, P.A. \& E.J. van \& de Jong, Y.S.D.M. (eds.). Fauna Europaea version 2.6, http://www.faunaeur.org.

Bordoni A., 1972 - Coleotterofauna dei muschi in un ambiente palustre di pianura. (Palude di Fucecchio, Toscana). Bollettino Associazione Romana di entomologia, 27 (1-2): 9-25.

Boriani M., Calvi M., Taddei A., Tantardini A., Cavagna B., Spadoni Andreani F., Montagna M., Bonini M., Lommen S., Müller-Schärer H., 2013 - Ophraella communa segnalata in Italia su Ambrosia. L'informatore Agrario, Verona, 34: 61-62.

Brivio C., 1970 - La coleotterofauna del Lago di Sartirana Briantea (Brianza Orientale, Lombardia). Estratto dalle Memorie della Società Entomologica Italiana, Genova, 49 (1970): 103-152.

Cannone N., 1997 - L'importanza delle praterie nel mantenimento della biodiversità a livello comunitario e gli impegni della Regione Lombardia. In: Atti del Seminario Permanente sulla gestione delle praterie e dei prati di interesse naturalistico, Monte Barro 25-26 ottobre 1996. Villa M. (ed.). Quaderni del Parco Monte Barro, 4 (1997): 89-102.

Cereda M., 2000 - Problematiche della gestione e prime azioni di conservazione dei prati magri nel Parco di Montevecchia e della Valle del Curone: il progetto Life Natura 1998 "Valle Santa Croce - Valle del Curone: tutela habitat prioritari". Quaderni del Parco Monte Barro, 5 (2000): 57-66.

Cereda M. \& Panseri E., 2003 - Il programma di gestione per le aree dei Festuco-Brometalia nel Parco di Montevecchia e Valle del Curone. Quaderni del Parco Monte Barro, 6 (2003): 23-40.

Daccordi M. \& Ruffo S., 2005 - Considerazioni biogeografiche sulle Chrysolina delle province appenninica e sicula con descrizione di Chrysolina (Stichoptera) bourdonnei n. sp. Studi Trentini Scienze Naturali. Acta Biologica, 81: 113-127.

Della Beffa G., 1911 - I Coleotteri dell'Agro torinese e loro rapporti colla vegetazione e l'agricoltura. Vincenzo Bona, Tipografia della Real Casa, Torino.

Doguet S., 1994 - Coléoptères Chrysomelidae, vol. 2: Alticinae. Faune de France. Fédération Française des Sociétés de Sciences Naturelles, Paris, 80: 1-696.

Fogato W. \& Leonardi C., 1980 - Coleotteri della Brughiera di Rovasenda (Piemonte). 1, la Brughiera pedemontana. Collana del Programma finalizzato "Promozione della qualità dell'ambiente", Roma: 25-73.

Fritzlar F., 2001 - Aphthona warchalowskii n. sp., Aphthona bergeali n. sp., Longitarsus angelikae n. sp. und Longitarsus meridionalis n. stat. 4 Blattkäfer (Coleoptera, Chrysomelidae, Alticinae) aus der südwestlichen Türkei. Entomologische Blätter für Biologie und Systematik der Käfer 97: 199-224.
Galibert H., 1932 - Dix-huit années de chasse aux Coléoptèeres dans les bassin de l'Agout (Tarn). Catalogue des espèces recueillies pendant cette période. Bulletin de la Société d'Histoire naturelle de Toulouse, 63: 27477.

Halbherr B., 1898 - Elenco sistematico dei Coleotteri finora raccolti nella Valle Lagarina. Museo Civico di Rovereto, Rovereto, 10.

Heikertinger F., 1912 - Biogeographische skizzen über palaearktischen Halticinen (Col.) (nebst. synonymischen Bemerkungen). Entomologische Mitteilungen, 1: 236-241; 383-388.

Heikertinger F., 1926 - Resultate fünfzehnjähriger Untersuchungen über die Nahrungspflanzen einheimischer Halticinen. Entomologische Blätter, 22:1-9; 49-62.

Heikertinger F., 1944 - Bestimmungstabelle der paläarktischen Aphthona, Arten. Koleopterologische Rundschau, 30 (1-3, 3-6): 37-124.

Holdhaus K., 1911 - Über die Coleopteren - und Molluskenfauna des Monte Gargano (unter besonderer Berücksichtigung der Adriatisfrage). Denkschriften der Mathematisch-Naturwissenschaftlichen Klasse der Kaiserlichen Akademie der Wissenschaften, 87: 431-465.

Konstantinov A.S. 1998 - Revision of the Palearctic species of Aphthona Chevrolat and cladistic classification of the Aphthonini (Coleoptera: Chrysomelidae: Alticinae). Associated Publishers, Gainesville.

Leonardi C. \& Doguet S., 1990 - Studio critico sui Longitarsus del gruppo pratensis (Panzer) (Coleoptera Chrysomelidae). Atti della Società italiana di Scienze naturali e del Museo civico di Storia naturale di Milano, 131 (2): 13-74.

Leonardi C. \& Sassi D. 1997 - I Crisomelidi (Coleoptera, Chrysomelidae) del Monte Barro. Memorie della Società Italiana di Scienze Naturali e del Museo Civico di Storia Naturale di Milano, Milano, 27 (2): 189227.

Löbl I. \& Smetana, A., 2010 - Catalogue of Palaearctic Coleoptera. Apollo Books, Stenstrup.

Luigioni P., 1929 - I Coleotteri d'Italia. Catalogo sinonimico, topografico-bibliografico. Memorie della Pontificia Accademia delle Scienze - I Nuovi Lincei, Roma, 13.

Müller G., 1953 - I Coleotteri della Venezia Giulia. Vol. II: Coleoptera Phytophaga (Cerambicidae, Chrysomelidae, Bruchidae), Centro sperimentale Agricoltura e Foreste, Trieste.

Panseri E., 2000 - Primo contributo alla conoscenza degli aspetti floristici e vegetazionali dei prati magri del Parco di Montevecchia e della Valle del Curone. In: Atti del Seminario Permanente sulla gestione delle praterie e dei prati di interesse naturalistico, Monte Barro, ottobre 2000. Villa M. (ed.). Quaderni del Parco Monte Barro, 5 (2000): 51-56.

Porta A., 1934 - Fauna Coleopterorum Italica. Vol. IV Heteromera, Phytophaga. Piacenza.

Previati, R. 1997 - Geologia. Origini, Evoluzioni e caratteristiche di un territorio. In: Parco di Montevecchia e della Valle del Curone. Bellavite Editore, Missaglia. 
Regalin R. 1981 - Le specie italiane del genere Labidostomis Redtenbacher (Coleoptera, Chrysomelidae, Clytrini). Estratto delle Memorie della Società Entomologica Italiana, Genova, 59.

Regalin R., Bezděk J., Penati F., Ciapponi L., 2006 - Catalogo topografico commentato dei Crisomelidi (Insecta, Coleoptera, Chrysomelidae) della Provincia di Sondrio (Lombardia, Italia settentrionale). Il Naturalista Valtellinese. Atti del Museo Civico di Storia Naturale di Morbegno, 17: 11-131.

Ronzoni, F.D. 1998 - Il Parco Regionale della Valle del Lambro. Bellavite Editore, Missaglia.

Sassi D. \& Borowiec L., 2006 - Cassida inopinata, a new species from Italy and Balkan Region (Coleoptera: Chrysomelidae: Cassidinae). Genus, Wroclaw, 17 (4): 545-560.

Sassi D., 2007 - I Crisomelidi (Coleoptera, Chrysomelidae) del Triangolo Lariano (Italia, Lombardia). Natura, Milano, 96 (2).

Thérond J., 1976 - Catalogues des Coléoptères de la Camargue et du Gard. Nîmes. Société d'étude des Sciences Naturelles, Mémoire, 10, $2^{\mathrm{e}}$ partie: 1-224.

Vigna Taglianti A., Audisio P.A., Biondi M., Bologna M.A., Carpaneto G.M., De Biase A., Fattorini S., Piattella E., Sindaco R., Venchi A., Zapparoli M., 1999 -A proposal for a chorotype classification of the Near East fauna, in the framework of the Western Palearctic region. Biogeographia, Lavori della Società Italiana di Biogeografia, (n.s.) 20: 31-59.

Weise J., 1888 - Chrysomelidae. In: Naturgeschichte der Insekten Deutschlands. Erste Abtheilung Coleoptera. Sechster Band. [1893]. Nicolaische Verlags - Buchandlung R. Stricker, Berlin: 769-960.

Zangheri P., 1969 - Repertorio sistematico e topografico della flora e della fauna vivente e fossile della Romagna. Tomo IV. Regno Animale. Da Coleoptera Phytophaga a Vertebrata e Fossili Plantae - Animalia. Memorie del Museo Civico di Storia Naturale di Verona, F.S., 1 (4): 1415-1962. 